hep-th/0507190

\title{
Profiling the Brane Drain in a Nonsupersymmetric Orbifold
}

\author{
Gregory Moore and Andrei Parnachev \\ Department of Physics, Rutgers University \\ Piscataway, NJ 08854-8019, USA
}

\begin{abstract}
We study D-branes in a nonsupersymmetric orbifold of type $\mathbb{C}^{2} / \Gamma$, perturbed by a tachyon condensate, using a gauged linear sigma model. The RG flow has both higgs and coulomb branches, and each branch supports different branes. The coulomb branch branes account for the "brane drain" from the higgs branch, but their precise relation to fractional branes has hitherto been unknown. Building on the results of hep-th/0403016 we construct, in detail, the map between fractional branes and the coulomb/higgs branch branes for two examples in the type 0 theory. This map depends on the phase of the tachyon condensate in a surprising and intricate way. In the mirror Landau-Ginzburg picture the dependence on the tachyon phase is manifested by discontinuous changes in the shape of the D-brane.
\end{abstract}

July 20, 2005 


\section{Introduction and summary}

An important property of string theory is that it is well-defined in the presence of certain spacetime singularities which render general relativity and quantum field theory ill-defined. Moreover, string theory contains mechanisms for smoothing out spacetime singularities. An interesting set of concrete examples of this phenomenon are spacetime nonsupersymmetric orbifolds of flat space [1]. (See [2] [3] for reviews). In such situations, the closed string spectrum contains tachyons whose wavefunctions are localized near the singular point; the resolution of singularities happens through the condensation of these tachyons. The presence of $\mathcal{N}=2$ worldsheet supersymmetry imposes constraints on the dynamics of the system, allowing one to understand the renormalization group (RG) flow [4] in a way analogous to the understanding of open string tachyon condensation (see e.g. [5]). In this paper we assume that the RG flow gives a good description of condensation of localized closed string tachyons. The behavior of the system in the IR corresponds to later times in the time evolution.

It is technically convenient to introduce the gauged linear sigma model (GLSM), whose higgs branch in the ultraviolet is the nonsupersymmetric orbifold [6][7]. In the process of RG flow, the higgs branch resolves into a smooth Hirzebruch-Jung space, which has a natural spacetime interpretation. The number of branes wrapping nontrivial two-cycles of the Hirzebruch-Jung space is generally smaller than that of fractional branes, so naively a "brane drain" is taking place. However one must bear in mind that the infrared theory contains a coulomb branch with isolated massive vacua. It has been suggested in [7] that Dbranes wrapping nontrivial cycles in the higgs branch, together with D-branes supported at the vacua of the coulomb branch, are in one-to-one correspondence with fractional branes in the orbifold theory. This picture has been sharpened in [8] where the open string Witten index was used to construct a map between the fractional branes and higgs and coulomb branch branes in the IR. 1 While [8] found the map in the case of type II string theory, a similar construction is possible in type 0 theory. We describe it in detail in Section 2.

One can study D-branes away from the conformal point using techniques developed in [12]. The mirror description of the GLSM is given by the Landau-Ginzburg (LG) theory with an inhomogeneous superpotential. Fractional branes localized at the orbifold fixed

1 The significance of the coulomb branch goes back to [9] 10] and was also recently emphasized in [11] where the correlators in the topologically twisted A-model were found to have support precisely on the coulomb branch. 
point preserve B-type supersymmetry, so they become A-type branes in the LG model. The latter are associated with critical points of the superpotential: the critical points away from the origin give rise to the coulomb branch branes, while those at the origin describe the higgs branch brane(s). In this paper we focus on the overlap of the boundary state with the identity operator (this is defined more precisely in Section 3). This quantity, which we call the generalized central charge, should in the first approximation be thought of as generalization of the D-brane mass to nonconformal theories. The generalized central charge of a brane described by the boundary state $|B\rangle$ can be computed as an integral

$$
\langle B \mid 1\rangle=\iint \frac{d x_{1}}{x_{1}} \frac{d x_{3}}{x_{3}} \exp (-W)
$$

over the A-brane surface. In (1.1) $x_{1}$ and $x_{3}$ are the LG fields, and $W$ is the superpotential. To determine the shape of the A-brane, it is necessary to solve certain soliton equations. The set of all solutions is parametrized by a small circle (wavefront) around the critical point. The A-brane surface is traced by the wavefront evolving in time [12].

We specialize to the case of $\mathbb{C}^{2} / \mathbb{Z}_{n(p)}, p=1$ orbifolds, whose minimal resolution contains a single non-trivial cycle. (The higgs branch in this case is $\mathcal{O}(-n) \rightarrow \mathbb{P}^{1}$.) There is a single higgs branch brane wrapping the nontrivial cycle, and $n-2$ coulomb branch branes associated with the massive vacua of the superpotential. We find that the generic form of the A-brane surface resembles that of a propeller. Near the critical point, the wavefront is a small circle, whose segments at late times trace various quarter-planes ("wings" of the "propeller"). This property can be used to compute (1.1) as a function of (complex) tachyon expectation value $w ; w \rightarrow 0$ corresponds to the orbifold (UV) limit, while $|w| \rightarrow \infty$ describes the IR regime. As explained in Section 4, (1.1) satisfies a GKZ equation, which in the Calabi-Yau case is a Picard-Fuchs equation for the periods. A basis of nonconstant solutions of the GKZ equation is given by the integrals (1.1) over the quarter planes. Linear combinations of these integrals, which we compute in Section 4, determine the generalized central charge of the A-brane whose wings asymptote to these quarter planes.

We analyze the behavior of (1.1) in the simple cases of $n=3,4$ in Section 5 . The intersection matrix and the quantum symmetry of the orbifold theory suffices to determine the map between the coulomb branch branes and the fractional branes. This map depends of the phase of the tachyon expectation value. Multiplication of $w$ by an $n$-th root of unity enforces the permutation symmetry of the fractional branes. In terms of the propeller surfaces, the asymptotics change discontinuously when the value of $w$ goes from one angular sector of the complex plane to another. 
As explained in Section 2, even after modding out by permutation symmetry of the fractional branes, in the type 0 theory there is more then one expression for the higgs branch brane which is consistent with the intersection matrix. In section 5 we find that each of the $n$ angular sectors which differ by a permutation of fractional branes is further divided into smaller subsectors, where different expressions for the higgs branch brane are realized. The generalized central charge for all higgs branch branes has the same leading logarithmic behavior in the regime of large $|w|$.

To summarize, this paper is organized as follows. In the next section we describe the orbifold, GLSM and its mirror LG model. We use the intersection matrix to relate fractional branes to the coulomb and higgs branch branes in the IR. In Section 3 we define the generalized central charge. In Section 4 we show that it solves the GKZ equation, and analyze the solutions. Section 5 is devoted to the detailed analysis of the $n=3$ and $n=4$ cases. We discuss our results and directions for future research in Section 6. Appendix A contains information on the construction of fractional brane boundary states. Appendix $\mathrm{B}$ is devoted to the numerical analysis of the shape of A-branes in the LG theory.

\section{Fractional branes vs. higgs and coulomb branch branes}

In this section we start by reviewing the results of [7] and [8] where the fate of fractional branes was studied using the gauged linear sigma model. We recall the mirror description in terms of the Landau-Ginzburg theory, and give a first hint at the appearance of the fractional branes in this language. We study the intersection form for type II and type 0 orbifolds and use it to construct the linear map between the fractional branes and the LG branes. We discuss the $\mathbb{C}^{2} / \mathbb{Z}_{3(1)}$ and $\mathbb{C}^{2} / \mathbb{Z}_{4(1)}$ examples in detail. Part of this section is review material. A more detailed exposition can be found in [4][7] [8] [2]. The map between the fractional branes and the LG (or, equivalently, GLSM) branes is spelled out in detail, although such a map was constructed implicitly in [8]. The discussion of the intersection form in the type 0 theory is new. The intersection form in type 0 theory is important for the $\mathbb{C}^{2} / \mathbb{Z}_{3(1)}$ example which is discussed at the end of this section and later in the paper. 


\subsection{Condensation of localized tachyons and the fate of the fractional branes.}

We consider type II or type 0 theory in $9+1$ dimensions. The orbifolding by $\mathbb{Z}_{n(p)}$ happens in the 67 and 89 planes, parametrized by complex coordinates $X^{(1)}$ and $X^{(2)}$. The orbifold group is generated by

$$
g=\exp \left(\frac{2 \pi i}{n}\left(J_{67}+p J_{89}\right)\right),
$$

where $J_{67}$ and $J_{89}$ generate rotations in two complex planes. When there are fermions in the theory, $p$ is defined $\bmod 2 n$. We will take the fundamental domain to be $p \in(-n, n)$. The action of $g^{n}$ on the Ramond sector ground state is a multiplication by $(-1)^{p \pm 1}$, depending on chirality. When $p$ is even, this acts as $(-1)^{F}$ where $F$ is the spacetime fermion number. In type II, there is no bulk tachyon and there are closed string fermions in the bulk, hence $p$ must be odd [1]. In the NSR formalism, a useful ingredient in the theory is the operator [4]

$$
X_{\frac{s}{n}}^{(i)}=\sigma_{s / n}^{(i)} \exp \left[i(s / n)\left(H^{(i)}-\tilde{H}^{(i)}\right)\right] ; \quad i=1,2 ; \quad s=1,2, \cdots, n-1
$$

where $H^{(i)}, \tilde{H}^{(i)}$ are the bosonised left- and right-moving fermions and $\sigma_{s / n}$ is the bosonic twist $s$ operator [13]. In the following we will restrict our attention to the left movers. There are two possible inequivalent choices for the worldsheet $\mathcal{N}=1$ supersymmetry generator in the theory. Correspondingly, there are two chiral rings which are BPS under these supersymmetries. The $(c, c)$ ring vertex operators are

$$
X_{\frac{s}{n}}^{(c c)}=X_{\frac{s}{n}}^{(1)} X_{\left\{\frac{s p}{n}\right\}}^{(2)},
$$

where $\{x\} \equiv x-[x]$ is the fractional part of $x$. The $(\mathrm{c}, \mathrm{a})$ ring vertex operators are

$$
X_{\frac{s}{n}}^{(a c)}=X_{\frac{s}{n}}^{(1)}\left(X_{1-\left\{\frac{s p}{n}\right\}}^{(2)}\right)^{*},
$$

The operation $p \rightarrow-p$ corresponds to exchanging the $(c, c)$ and the $(c, a)$ rings. Therefore we can restrict ourselves to the theories with $p \in(0, n)$.

The generators of the $(\mathrm{c}, \mathrm{a})$ ring, denoted $W_{\alpha}, \quad \alpha=1 \ldots r$ form a collection of (in general) relevant operators. Turning these on in the action induces RG flow to the minimal

resolution of the singularity [4, ]1. For the $\mathbb{C}^{2} / \mathbb{Z}_{n(p)}$ orbifold such a resolution is encoded in the continued fraction

$$
\frac{n}{n-p}=a_{1}-\frac{1}{a_{2}-\frac{1}{a_{3}-\ldots}}:=\left[a_{1}, a_{2}, \ldots a_{r}\right],
$$


Note the appearance of $n-p$ rather then $p$ in (2.5), since we are talking about the $(\mathrm{c}, \mathrm{a})$ ring. The smooth space which appears after the minimal resolution of the singularity is called the Hirzebruch-Jung manifold. The generators of the chiral ring are in one-to-one correspondence with the exceptional $\mathbb{P}^{1}$ 's of this space. Their intersection numbers are given by

$$
C_{\alpha \beta}=-\delta_{\alpha, \beta-1}+a_{\alpha} \delta_{\alpha, \beta}-\delta_{\alpha, \beta+1}
$$

The (c,c) ring generators give rise to the resolution with similar properties; one needs to substitute $n-p \rightarrow p$ in (2.5). In the type II theory one should bear in mind the existence of a chiral GSO projection. As explained in [8], all generators $W_{\alpha}$ in the $(\mathrm{c}, \mathrm{a})$ ring survive the GSO projection if and only if all $a_{j}$ are even integers. In the $(\mathrm{c}, \mathrm{c})$ ring at least one generator is projected out [8]. In the type 0 theory chiral operators in $(c, a)$ and $(c, c)$ rings are not projected out by the diagonal GSO projection. Since $p \rightarrow p+n$ does not affect the ring structure, but only affects the GSO projection, the closed string sector in type 0 theories is unchanged under this operation. This means that type 0 theories with $p$ and $n-p$ are isomorphic: they are related by interchanging the (c,c) and the (c,a) rings. Put differently, in a type 0 theory with a given $p$, one should be able to resolve the singularity into two different Hirzebruch-Jung spaces, whose intersection numbers correspond to continued fractions determined by both $p$ and $n-p$. In [8] it has been shown that branes in type II theory which wrap nontrivial two-cycles in the Hirzebruch-Jung space are given by linear combinations of the fractional branes. In this paper we will see that the situation in the type 0 string theory is similar. Since there are two different resolutions, one can define two sets of branes wrapping the two-cycles in these spaces. Both sets are given by certain linear combinations of fractional branes.

In [7] the gauged linear sigma model (GLSM) was used to shed light on the fate of fractional D-branes in the process of twisted tachyon condensation. The field content of the relevant GLSM involves $r$ abelian $\mathcal{N}=2$ gauge fields $V_{\alpha}, \alpha=1, \ldots, r$ coupled to $r+d$ $\mathcal{N}=2$ chiral matter fields $X_{i}$ with charges

$$
Q_{\alpha i}=-C_{\alpha i}
$$

with $C_{\alpha i}$ given by [compare with (2.6)]:

$$
C_{\alpha i}=-\delta_{\alpha, i-1}+a_{\alpha} \delta_{\alpha, i}-\delta_{\alpha, i+1} ; \quad i=0, \ldots, r+1
$$


The field strengths of the gauge fields are contained in twisted chiral superfields $\Sigma=$ $\frac{1}{2}\left\{\overline{\mathcal{D}}, \mathcal{D}^{*}\right\}$. The classical Lagrangian is

$$
\mathcal{L}=\int d^{4} \theta\left(\bar{X}_{i} e^{2 Q_{\alpha i} V_{\alpha}} X_{i}-\frac{1}{2 e_{\alpha}^{2}} \bar{\Sigma}_{\alpha} \Sigma_{\alpha}\right)-\frac{1}{2}\left(\int d^{2} \tilde{\theta} t_{\alpha} \Sigma_{\alpha}+\text { c.c. }\right),
$$

where repeated indices are summed and

$$
t_{\alpha}=\zeta_{\alpha}-i \theta_{\alpha}
$$

combines the Fayet-Iliopoulos (FI) parameter $\zeta$ and theta angle $\theta$ for the $\alpha^{\text {th }}$ gauge field; $d^{2} \tilde{\theta}$ is the twisted chiral superspace measure. The renormalized FI parameters at the scale $\mu$ is

$$
t_{\alpha, \mathrm{eff}}(\mu)=t_{\alpha, \mathrm{bare}}+\sum_{i=1}^{r+d} Q_{\alpha i} \log \frac{\mu}{\Lambda}
$$

where $t_{\alpha, \text { bare }}$ are bare parameters defined at the momentum cutoff scale $\Lambda$. Due to (2.7), (2.6), $t_{\alpha, \text { bare }} \rightarrow-\infty$ in the UV. As explained in [7], in this regime the higgs branch describes the $\mathbb{C}^{2} / \mathbb{Z}_{n(p)}$ orbifold. The theory also contains a coulomb branch, where the lowest components of $\Sigma_{\alpha}$ get expectation values. In the infrared, the higgs branch becomes the Hirzebruch-Jung space [7]. We will call branes wrapping two-cycles in this resolved space "higgs branch branes". Another important property of the infrared physics is decoupling of the coulomb branch from the higgs branch. The former develops a set of massive isolated minima. Some fractional branes become B-branes "supported" at these coulomb branch vacua, as twisted tachyons condense [7]. We call such branes "coulomb branch branes".

For our purposes it will be more convenient to look at the "mirror" description of the coulomb branch that follows from the approach to mirror symmetry using abelian duality of 2D gauge theory of Morrison and Plesser [10] 114. This can be cast in terms of an effective Landau-Ginzburg theory [12]. (We follow the line of argument explained in [7].) The chiral superfields $X^{i}$ are eliminated in favor of the twisted scalar superfields $Y_{i}$. The twisted superpotential in the theory reads

$$
\widetilde{W}=\sum_{\alpha=1}^{r} \Sigma_{\alpha}\left(\sum_{i=0}^{r+1} Q_{\alpha i} n Y_{i}-t_{\alpha}(\mu)\right)+\mu \sum_{i} \lambda_{i} e^{-n Y_{i}}
$$

Integrating out the $Y_{i}$ gives the effective superpotential of [9] [10], while eliminating instead the $\Sigma_{\alpha}$ and $Y_{\alpha} \alpha=1, \ldots, r$, gives (in terms of $u_{0}=\left(\mu \lambda_{0}\right)^{1 / n} \exp \left[-Y_{0}\right]$ and $\left.u_{r+1}=\left(\mu \lambda_{r+1}\right)^{1 / n} \exp \left[-Y_{r+1}\right]\right)$

$$
\widetilde{W}=u_{0}^{n}+u_{r+1}^{n}+\sum_{\alpha=1}^{r} \lambda_{\alpha}^{\prime} u_{0}^{p_{\alpha}} u_{r+1}^{q_{\alpha}}
$$


where

$$
\lambda_{\alpha}^{\prime}=\lambda_{\alpha} \Lambda^{1-\Delta_{\alpha}} e^{t_{\alpha, \mathrm{bare}}^{\prime}}=\lambda_{\alpha} \mu^{1-\Delta_{\alpha}} e^{t_{\alpha, \mathrm{eff}}^{\prime}(\mu)}
$$

The scaling dimensions of the $\alpha$ 's operator in the sum identifies it with the ring generator $W_{\alpha}$. Two important comments are in order

(1) The LG description involves dualizing the phases of $X_{i}$, and hence is not well defined near the higgs branch, where $X_{\alpha}=0$. We do expect it to give a correct description of the coulomb branch though. As we will see later in the paper, we will be able to recover the description of the higgs branch as well. The essential ingredient will be the identification of the higgs branch brane with a combination of the fractional branes with the help of intersection form.

(2) The 'mirror' $\mathbb{Z}_{n}$ transformation

$$
\left(u_{0}, u_{r+1}\right) \sim\left(\omega u_{0}, \omega^{-p} u_{r+1}\right)
$$

leaves the effective superpotential (2.13) invariant - it fixes all the $\Sigma_{\alpha}^{\prime}$. Indeed it is a gauge symmetry remnant of the duality transformation and therefore we should quotient the LG model by its action.

In this paper we will be concerned with the simplest case of a continued fraction of length one. This corresponds to $p=1, r=1, a_{1}=n$. There are two fields, $x_{1} \equiv u_{0}$ and $x_{3} \equiv u_{2}$. The tachyon expectation value is determined by the parameter $w \equiv \lambda_{1}^{\prime}$. The LG superpotential is

$$
W=x_{1}^{n}+w x_{1} x_{3}+x_{3}^{n}
$$

and the theory should be quotiented by

$$
\left(x_{1}, x_{3}\right) \sim\left(e^{\frac{2 \pi i}{n}} x_{1}, e^{-\frac{2 \pi i}{n}} x_{3}\right)
$$

\subsection{Intersection form and the map between $L G$ and fractional branes}

We start by reviewing the results of [8]. There we considered the D-brane intersection form in type II string theory on the orbifold $\mathbb{C}^{2} / \Gamma[15-17$

$$
\mathcal{I}_{a b}=\operatorname{tr}_{R, a b}(-1)^{F} q^{L_{0}-\frac{c}{24}}
$$


Here the trace is over the states of the open string suspended between D-branes which correspond to representations of $\Gamma$ labeled by $a$ and $b$ and $F$ is the worldsheet fermion number. In the case of type II theory, this formula can be written as [8]

$$
\mathcal{I}_{a b}=\frac{4}{n} \sum_{s=0}^{n-1} \exp \left(\frac{2 \pi i(b-a) s}{n}\right) \sin \left(\frac{\pi s}{n}\right) \sin \left(\frac{\pi s p}{n}\right)
$$

The first factor in the sum comes from the action of the group element on the Chan-Phaton factors, while the product of the sin's is due to the fermion zero modes in the $\mathrm{R}$ sector. It is not hard to evaluate 2.19$)$ :

$$
\mathcal{I}_{a b}=\delta_{a-b-\frac{1-p}{2}}+\delta_{a-b+\frac{1-p}{2}}-\delta_{a-b-\frac{1+p}{2}}-\delta_{a-b+\frac{1+p}{2}}
$$

where

$$
\delta_{a} \equiv \delta_{a, 0 \bmod n}
$$

Note that the arguments of delta functions in (2.20) are always integers, thanks to the requirement that $p$ is odd. The matrix $\mathcal{I}$ in 2.20 is written in the basis

$$
e_{0}, e_{1}, \ldots, e_{n-1}
$$

where $e_{a}$ is the $a$-th fractional brand2. The intersection matrix $\mathcal{I}$ is invariant under the cyclic permutation of fractional branes, $e_{a} \rightarrow e_{a+1}$. In [8] we found a change of basis which block-diagonalizes $\mathcal{I}$; one of the two blocks is given precisely by the intersection matrix for the higgs branch branes $C_{\alpha \beta}$. However this block-diagonalization is clearly invariant under the cyclic permutation of the fractional branes; in other words, if certain expressions for the higgs branch branes

$$
h_{\alpha}=\sum_{a} H_{\alpha a} e_{a}, \quad H_{\alpha a} \in \mathbb{Z}
$$

give rise to the intersection matrix $C_{\alpha \beta}$, then the expressions with the indices of fractional branes permuted,

$$
h_{\alpha}=\sum_{a} H_{\alpha a} e_{a+1}
$$

are equally good. We will see later in the paper that, thanks to the dependence of the map between fractional and coulomb/higgs branch branes on the tachyon VEV, all possible

2 The details of fractional brane construction in type 0 and type II string theories are summarized in Appendix A. 
cyclic permutation of fractional branes are realized, depending on the phase of the tachyon expectation value. For now we assume the basis (2.22) for simplicity, keeping the permutation symmetry in mind. In fact, it is convenient to switch to the basis $\sum_{a} e_{a}, e_{1}, \ldots, e_{n-1}$ which effectively substitutes both the first row and the first column in $\mathcal{I}$ by a set of zeroes (since the D0 brane, $D 0=\sum_{a} e_{a}$, has zero intersection with any brane in the theory, including itself). Now we can omit the first row and column from $\mathcal{I}$ - it is this reduced matrix, denoted $\tilde{\mathcal{I}}$ which appears in the rest of this paper, unless stated otherwise.

We would like to generalize this discussion for type 0 . The number of branes is now doubled, for there are branes which are labeled by the choice of sign in the gluing conditions (see Appendix A for details). We will call these branes $\eta=+1$ or $\eta=-1$ branes3. As explained in [8], when $p$ is odd, the intersection form of the type 0 theory is simply obtained from the intersection form $\tilde{\mathcal{I}}$ of type II:

$$
\tilde{\mathcal{I}}_{0}=\left(\begin{array}{cc}
0 & \tilde{\mathcal{I}} \\
\tilde{\mathcal{I}}^{T} & 0
\end{array}\right)
$$

where $\tilde{\mathcal{I}}^{T}=\tilde{\mathcal{I}}$. Note that fermionic degrees of freedom only exist on the intersections of branes of different types. It will be convenient to separate $\eta=+1$ and $\eta=-1$ fractional branes into two sets: the ones that are labeled by the "special representation" integer $e_{p_{\alpha}}$ and the rest, $e_{\nu}$. The integers $p_{\alpha}$ are determined by the continued fraction $\left[a_{1}, a_{2}, \ldots a_{r}\right]$ via the recursion relations [7]:

$$
p_{j-1} / p_{j}=\left[a_{j}, a_{j+1}, \ldots, a_{r}\right], \quad 1 \leq j \leq r
$$

with the initial conditions $p_{r+1}=0, p_{r}=1$. In the basis

$$
e_{p_{1}}^{(+)}, \ldots, e_{p_{r}}^{(+)},\left\{e_{\nu}^{(+)}\right\}, e_{p_{1}}^{(-)}, \ldots, e_{p_{r}}^{(-)},\left\{e_{\nu}^{(-)}\right\}
$$

we consider the following ansatz for the linear map between the LG branes (higgs and coulomb branch branes $h_{\alpha}$ and $c_{\alpha}$ ) and the fractional branes

$$
\left(\begin{array}{c}
h_{\alpha}^{(+)} \\
c_{\alpha}^{(+)} \\
h_{\alpha}^{(-)} \\
c_{\alpha}^{(-)}
\end{array}\right)=\left(\begin{array}{cc}
A & 0 \\
0 & B
\end{array}\right)\left(\begin{array}{c}
e^{(+)} \\
e^{(-)}
\end{array}\right)=\left(\begin{array}{cccc}
1 & a & 0 & 0 \\
0 & 1 & 0 & 0 \\
0 & 0 & 1 & b^{T} \\
0 & 0 & 0 & 1
\end{array}\right)\left(\begin{array}{c}
e_{p_{\alpha}}^{(+)} \\
e_{\nu}^{(+)} \\
e_{p_{\alpha}}^{(-)} \\
e_{\nu}^{(-)}
\end{array}\right)
$$

3 These branes are often called electric and magnetic branes in the literature, but they are not electric/magnetic duals. In type 0 theories obtained as orbifolds by $(-1)^{F_{\text {spacetime }}}$ of the type II string, they are in fact fractional branes 8$]$. 
where $a$ and $b$ are matrices whose entries are integers. We then require

$$
\left(\begin{array}{cc}
A & 0 \\
0 & B
\end{array}\right)\left(\begin{array}{cc}
0 & \tilde{\mathcal{I}} \\
\tilde{\mathcal{I}}^{T} & 0
\end{array}\right)\left(\begin{array}{cc}
A^{T} & 0 \\
0 & B^{T}
\end{array}\right)=\left(\begin{array}{cccc}
0 & 0 & C & 0 \\
0 & 0 & 0 & C^{\prime} \\
C & 0 & 0 & 0 \\
0 & C^{\prime} & 0 & 0
\end{array}\right)
$$

One can solve for $a, b$ and $C$. The result is

$$
a=-x C^{\prime-1}, \quad b=-C^{\prime-1} y, \quad C=C_{1}-x C^{\prime-1} y
$$

where $C_{1}, C^{\prime}, x$ and $y$ are the components of $\tilde{\mathcal{I}}$ :

$$
\tilde{\mathcal{I}}=\left(\begin{array}{cc}
C_{1} & x \\
y & C^{\prime}
\end{array}\right)
$$

Note that the map determined by $(2.30)$ is determined up to an addition/subtraction of any multiple of D0 branes, since the latter have zero intersection with any fractional brane.

When $p$ is odd, the orbifold group is $\mathbb{Z}_{n}$, one can define type II theory, and $\tilde{\mathcal{I}}=\tilde{\mathcal{I}}^{T}$. In this case $\tilde{\mathcal{I}}$ is given by the reduction of (2.20), and $a=b^{T}$. In 8 ] it was shown that if in addition all $a_{\alpha}$ are even, then the entries of $a$ (and $b$ ) in (2.30) are integers and $C$ computed in (2.30) coincides with (2.6). The closed string sector of type 0 theory is invariant under $p \rightarrow p+n$. However eq. 2.19) is not invariant under such a shift. This is a manifestation of the fact that one can define two sets of branes in the type 0 theory. These two types of branes will have intersection matrices corresponding to two different types of resolution, as discussed above. A simple set of examples considered in [8] is $p=1, n-1$. The intersection form for the $p=1$ case reproduces the Cartan matrix which defines the supersymmetric ALE singularity. For $p=n-1$ there is a single higgs branch brane $h$ which wraps the base of $\mathcal{O}(-n) \rightarrow \mathbb{P}^{1}$ which is the Hirzebruch-Jung manifold in this case. The change of basis in the $p=n-1$ case is nontrivial; here we quote the result obtained in $[8]$ :

$$
h=e_{1}+2 e_{2}+\ldots+\frac{n}{2} e_{\frac{n}{2}}-\left(\frac{n}{2}-1\right) e_{\frac{n}{2}+1}-\ldots-e_{n-1}
$$

Suppose now $p$ is even. (And, consequently, $n$ is odd as we restrict our consideration to $n, p$ relatively prime.) In this case the orbifold group is $\mathbb{Z}_{2 n}$ and expression (2.19) for the unreduced matrix $\mathcal{I}_{0}$ should be modified to

$$
\mathcal{I}_{0, a b}=\frac{4}{2 n} \sum_{s=0}^{2 n-1} \exp \left(\frac{2 \pi i(a-b) s}{2 n}\right) \sin \left(\frac{\pi s}{n}\right) \sin \left(\frac{\pi s p}{n}\right)
$$


The indices $a, b$ which label the branes now run from 0 to $2 n-1$. $n$ is substituted by $2 n$ in the prefactor and in the first factor in the sum- this is the result of the order of the orbifold group becoming $\mathbb{Z}_{2 n}$. The matrix element in (2.33) evaluates to zero whenever $a-b$ is even. This is an indication that $a, b$ now label not only different types of fractional branes, but also $\eta=+1$ and $\eta=-1$ types. We can define $a=2 a^{\prime}$ and $b=2 b^{\prime}+1$ with $a^{\prime}=0, \ldots, n-1$ and $b^{\prime}=0, \ldots, n-1$ labeling $\eta=+1$ and $\eta=-1$ branes respectively. The intersection form is then of the form (2.25), although $\mathcal{I}$ is no longer a symmetric matrix:

$$
\mathcal{I}_{a^{\prime} b^{\prime}}=\delta_{a^{\prime}-b^{\prime}+\frac{p}{2}}+\delta_{a^{\prime}-b^{\prime}+1-\frac{p}{2}}-\delta_{a^{\prime}-b^{\prime}-\frac{p}{2}}-\delta_{a^{\prime}-b^{\prime}+1+\frac{p}{2}}
$$

To use (2.30) we still need to factor out the $D 0$ brane by omitting the first row and the first column. At this point it is worth mentioning the following important issue that was not present in the type II theory. We can shift the $\eta=-1$ branes by $e_{a^{\prime}}^{(-)} \rightarrow e_{a^{\prime}+1}^{(-)}$, without shifting the $\eta=+1$ branes. This leads to permutation of columns, $\mathcal{I}_{a^{\prime}, b^{\prime}} \rightarrow \mathcal{I}_{a^{\prime}, b^{\prime}+1}$. After the reduction to the subspace which does not contain a D0 brane, we obtain $n$ inequivalent matrices $\tilde{\mathcal{I}}$ this way. In this paper we mostly discuss the $p=1$ case for $n=3,4$. As

explained in Section 5, all inequivalent matrices $\tilde{\mathcal{I}}$ obtained as described above, admit a block-diagonalization of the form (2.29). The analysis for general $n$ and $p$ will be reported elsewhere.

\section{D-branes in the LG mirror and generalized central charges}

The superpotential of the LG model providing a mirror description of the GLSM (2.9) has a set of critical points. According to [12] each critical point gives rise to a D-brane with A-type boundary conditions. This D-brane, which we will sometimes refer to as an A-brane surface, is a Lagrangian submanifold of $\mathbb{C}^{2}$ whose image in the $W$-plane is a half-line which starts at the critical value and extends in the positive real direction. The critical points away from $\left(x_{1}, x_{3}\right)=(0,0)$ correspond to the coulomb branch branes, while the single critical point at the origin gives rise to a higgs branch brane (recall that we specialize to the case of continued fraction of length one)

As explained in [12] a practical way to determine these surfaces is to consider the soliton equation

$$
\frac{d x^{i}}{d \sigma}=\frac{1}{2} g^{i \bar{j}} \partial_{\bar{j}} \bar{W}
$$

where $g_{i \bar{j}}$ is the Kahler metric and $\sigma$ is the coordinate along the soliton trajectory. The soliton trajectory is supposed to originate from a critical point, where the Kahler metric 
is nonsingular. (This leads to problems when considering the higgs branch brane, whose critical point is at $\left(x_{1}, x_{3}\right)=(0,0)$ where the Kahler metric cannot even be reliably determined). The shape of the A-brane associated with a given critical point is the set of all trajectories satisfying (3.1). Near the critical point this shape can be easily determined. One needs to find the coordinates $u^{i}$ which diagonalize the system (3.1). In these coordinates, the solutions are $u^{i}=u_{0}^{i} \exp \left(\lambda^{i} \sigma\right)$, so that $u^{i} \rightarrow 0$ as $\sigma \rightarrow-\infty$. Consistency of the equations (3.1) then forces $u_{0}^{i} \in \mathbb{R}$. A convenient way of parameterizing a set of solutions [12] is considering a sphere of small radius $\epsilon$

$$
\sum_{i}\left(u^{i}\right)^{2}=W\left(\phi_{*}^{i}+u^{i}\right)-W\left(\phi_{*}^{i}\right)=\epsilon^{2}, \quad u^{i} \in \mathbb{R}
$$

As $\sigma$ increases, this small sphere ("the wavefront") will evolve; the surface traced by it in this process is the A-brane. In Appendix B we analyze these surfaces numerically. We find that in the models we consider they resemble a propeller, with various segments of the small circle developing into quarter-plane "wings".

An interesting object that one can consider in the LG models is an overlap of a RR ground stat 1 , corresponding to the identity operator, with the D-brane specified by the boundary state $|B\rangle$ [12]. To make this object holomorphic, one needs to consider the formal limit $\bar{W} \rightarrow 0$ [12]. In this case, the overlap can be computed as an integral over the A-brane surface:

$$
\langle B \mid 1\rangle=\iint \frac{d x_{1}}{x_{1}} \frac{d x_{3}}{x_{3}} \exp (-W)
$$

As we will see in the next section, this integral is convergent, since $W \rightarrow+\infty$ in the asymptotic region. Moreover, in the next section we will also see that (3.3) satisfies the GKZ equation. In the supersymmetric case the solutions would give rise, via mirror symmetry, to the integrals of the complexified Kahler form over the cycles in the higgs branch of the GLSM. This motivates us to call the quantity (3.3) "the generalized central charge" of the D-brane described by the boundary state $|B\rangle$. In the non-Calabi-Yau case of this paper, the higgs branch brane in the GLSM still has a geometric interpretation of a brane wrapping a two-cycle in the Hirzebruch-Jung space.

Note that the branes described above correspond to the B-branes in the original GLSM and orbifold theories with $\eta=1$. Before performing the GSO projection, the theory

4 The correspondence is realized by performing the worldsheet path integral on the semiinfinitely long cigar with no insertions, but in the twisted theory, to produce a RR state. 
contains a second set of branes with $\eta=-1$, which preserve a different combination of the worldsheet $\mathcal{N}=2$ supersymmetry. Such branes are described by eq. (3.1) with an additional minus sign in the right-hand side. The image of these branes in the $W$-plane would therefore be half-lines extending in the negative real direction. The definition (3.3) would have to be modified accordingly.

\section{Generalized periods for the $\mathbb{C}^{2} / \mathbb{Z}_{n(1)}$ orbifold}

In this section we consider the generalized periods for the LG superpotential

$$
W:=a_{1} x_{1}^{n}+a_{2} x_{1} x_{3}+a_{3} x_{3}^{n}
$$

This section is rather technical. A summary is found in the final subsection.

\subsection{GKZ Equation}

Associated to a toric manifold is a canonically determined system of differential equations, the GKZ system of differential equations. For toric hypersurfaces in CY manifolds these equations are related to the Picard-Fuchs equations of the mirror and therefore solutions are related to the periods of the mirror variety.

In the present case, using the toric data of the $\mathbb{C}^{2} / \mathbb{Z}_{n(1)}$ manifold one finds the differential equation

$$
\left[\Theta^{2}-z(n \Theta)(n \Theta+1) \cdots(n \Theta+n-1)\right] F(z)=0
$$

with $\Theta=z \frac{d}{d z}$. $z=\infty$ is a regular singular point, and corresponds to the orbifold point. $z=0$ is an irregular singular point and corresponds to the "IR limit."

To derive (4.2) one begins with the fan $v_{1}=(0,1), v_{2}=(1,0), v_{3}=(n,-1)$ to produce the differential operator

$$
\mathcal{D}:=\left(\partial_{a_{1}} \partial_{a_{3}}-(-1)^{n} \partial_{a_{2}}^{n}\right)
$$

Now, again using the toric vectors one defines an invariant combination $z:=a_{1} a_{3} / a_{2}^{n}$. When acting on a function depending on $a_{i}$ only through $z$ it is straightforward to show that

$$
\mathcal{D} f(z)=\frac{1}{a_{1} a_{3}}\left[\Theta^{2}-z(n \Theta)(n \Theta+1) \cdots(n \Theta+n-1)\right] F(z)
$$

This establishes (4.2). 
A useful change of variable $w=z^{-1 / n}=a_{2} /\left(a_{1} a_{3}\right)^{1 / n}$ brings the GKZ equation to the form:

$$
\left[-\left(-\frac{d}{d w}\right)^{n}+\frac{1}{n^{2}}\left(w \frac{d}{d w}\right)^{2}\right] f(w)=0
$$

The $n$-dimensional space of solutions to this equation is the $n$-dimensional space of generalized periods. The constant solution - which will be associated with the $D 0$ brane - is somewhat trivial and we define $\mathcal{V}$ to be the space of nonconstant solutions which vanish at $w=0$. Much of the work in Section 5 of this paper will be writing down different bases for

$\mathcal{V}$ and interpreting them physically. One basis of solutions is obtained by straightforward application of the Frobenius technique. This is:

$$
\begin{aligned}
\hat{f}_{-1}(w) & :=1 \\
\hat{f}_{m}(w) & :=(-w)^{m+1} \sum_{k=0}^{\infty}(-w)^{n k} \frac{\left(\Gamma\left(k+\frac{m+1}{n}\right)\right)^{2}}{\Gamma(k n+m+2)} \quad 0 \leq m \leq n-2
\end{aligned}
$$

Note that $\hat{f}_{m}(w)$ are entire functions in the $w$-plane for $n>2$ with an essential singularity at $w=\infty$. It will be useful to extend the definition (4.6) to include $\hat{f}_{n-1}(w)$, although this function does not solve the GKZ equation.

\subsection{Integral representation}

In the physical interpretation of the solutions it is very useful to have an integral representation directly related to the path integral which computes the overlap between RR groundstates and the branes. This solution has the schematic form

$$
\int_{\gamma} e^{-W} \frac{d x_{1}}{x_{1}} \frac{d x_{3}}{x_{3}}
$$

where $\gamma$ is an appropriate contour, to be discussed in detail below.

Note that it is trivially true that for $W$ given by (4.1) and $\mathcal{D}$ given by (4.3)

$$
\mathcal{D} \int_{\gamma} e^{-W} \frac{d x_{1}}{x_{1}} \frac{d x_{3}}{x_{3}}=0
$$

where $\gamma$ is any fixed chain of real dimension 2 in $\mathbb{C}^{*} \times \mathbb{C}^{*}$.

Now, the generalized periods should only be functions of the scaling variable $w$. This can be arranged by exploiting the $\mathbb{C}^{*} \times \mathbb{C}^{*}$ action on $\left(x_{1}, x_{3}\right)$. In fact, by analyticity, we need only consider $\mathbb{R}_{+}^{*} \times \mathbb{R}_{+}^{*}$ invariant orbits. Any such orbit through $(\alpha, \beta) \in \mathbb{C}^{*} \times \mathbb{C}^{*}$ is of the form

$$
\gamma_{\alpha, \beta}:=\left\{\left(t_{1} \alpha, t_{3} \beta\right) \mid t_{1}, t_{3}>0\right\} .
$$


One might be tempted to use the chains $\gamma_{\alpha, \beta}$ in (4.7) to produce solutions of (4.5). There are two problems with this. First, we must also regularize the logarithmic singularities at the origin. Next, we must ensure convergence of the integral at $\infty$. The singularity at the origin is easily regularized by taking $t_{i} \geq \epsilon$. Thus we consider the contour integral:

$$
I_{\alpha, \beta}(w ; \epsilon):=\int_{\epsilon}^{\infty} \frac{d t_{1}}{t_{1}} \int_{\epsilon}^{\infty} \frac{d t_{3}}{t_{3}} e^{-W}
$$

where

$$
W=\alpha^{n} t_{1}^{n}+\alpha \beta w t_{1} t_{3}+\beta^{n} t_{3}^{n}
$$

with $t_{1}, t_{3}>0$ always.

We will use this basic integral to construct solutions. To ensure convergence at infinity we require $\operatorname{Re}\left(\alpha^{n}\right)>0$ and $\operatorname{Re}\left(\beta^{n}\right)>0$. There are different sectors of the $\left(x_{1}, x_{3}\right)$ plane in which the integrals converge. We will need a way of denoting these convergent sectors. Define $\alpha=|\alpha| e^{i \theta}$. Then if $\alpha$ is in a convergent sector there must exist an integer $s_{\alpha}$ such that

$$
-\frac{\pi}{2 n}<\theta+\frac{2 \pi}{n} s_{\alpha}<\frac{\pi}{2 n}
$$

We will denote the convergent sectors (4.12) in the $\alpha$ plane by $\mathcal{S}_{s_{\alpha}}$. Equation (4.12) only defines $s_{\alpha}$ modulo $n$. If we choose the principal branch of the logarithm then $s_{\alpha}$ is defined absolutely. We choose the fundamental domains: 目

$$
\begin{aligned}
s & =\frac{n-1}{2}, \frac{n-3}{2}, \ldots,-\frac{n-1}{2} \quad n \text { odd } \\
s & =\frac{1}{2} n, \ldots,-\frac{1}{2} n \quad n \text { even }
\end{aligned}
$$

See Figs. 1,2 for $n=3,4$.

By using rescalings of $t_{1}, t_{3}$, the equation $\mathcal{D} e^{-W}=0$, and keeping track of boundary terms, and (4.4) it is straightforward to show that

$a_{1} a_{3} \mathcal{D} I_{\alpha, \beta}(w ; \epsilon)=\frac{1}{n^{2}} e^{-W\left(t_{1}=\epsilon, t_{3}=\epsilon\right)}-\frac{\alpha^{n}}{n} \int_{\epsilon}^{\infty} d t_{1} t_{1}^{n-1} e^{-W\left(t_{1}, t_{3}=\epsilon\right)}-\frac{\beta^{n}}{n} \int_{\epsilon}^{\infty} d t_{3} t_{3}^{n-1} e^{-W\left(t_{1}=\epsilon, t_{3}\right)}$

5 This has one awkward feature for $n$ even: In this case the angular sector containing the negative real axis is a convergent sector and $s$ is discontinuous across the negative real axis, jumping from $s=-n / 2$ just above the negative real axis to $s=n / 2$ just below the negative real axis. 


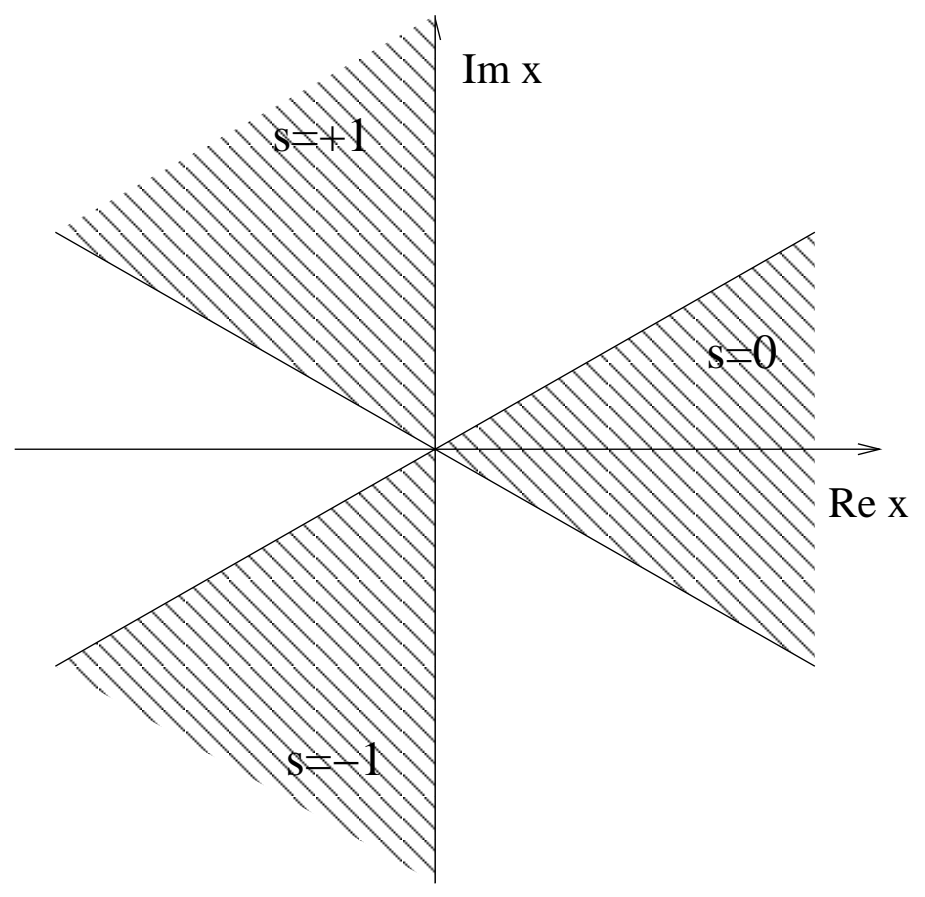

Fig 1. The angular structure in the $\alpha, \beta$ (equivalently, $x_{1}, x_{3}$ ) planes for $n=3$. In the shaded sectors the integral (4.10) converges.

Thus, the $I_{\alpha, \beta}(w ; \epsilon)$ do not solve the differential equation. Note, however, that the RHS of (4.14) has a smooth $\epsilon \rightarrow 0$ limit, given by $-1 / n^{2}$

We will form solutions from the $I_{\alpha, \beta}$ by taking appropriate linear combinations of $I_{\alpha, \beta}(w ; \epsilon)$ and taking the $\epsilon \rightarrow 0$ limit. In order to produce appropriate combinations let us investigate the $\epsilon \rightarrow 0$ behavior. Note that $I_{\alpha, \beta}(w ; \epsilon)$ has a convergent expansion in $w$. If we expand the integrand in (4.10) in a power series in $w$, only the first term has a divergence as $\epsilon \rightarrow 0$. Let us define $\hat{I}_{\alpha, \beta}(w ; \epsilon)$ via:

$$
I_{\alpha, \beta}(w ; \epsilon)=\int_{\epsilon}^{\infty} \frac{d t_{1}}{t_{1}} e^{-\alpha^{n} t_{1}^{n}} \int_{\epsilon}^{\infty} \frac{d t_{3}}{t_{3}} e^{-\beta^{n} t_{3}^{n}}+\hat{I}_{\alpha, \beta}(w ; \epsilon)
$$

The first term is divergent for $\epsilon \rightarrow 0$, and constant in $w$. In fact, one has:

$$
\int_{\epsilon}^{\infty} \frac{d t_{1}}{t_{1}} e^{-\alpha^{n} t_{1}^{n}}=\frac{1}{n} E_{1}\left(\epsilon^{n} \alpha^{n}\right)=-\log \epsilon-\frac{1}{n} \log \alpha^{n}-\frac{\gamma}{n}+\mathcal{O}\left(\epsilon^{n}\right) .
$$

Thus we conclude that

$$
I_{\alpha, \beta}(w ; \epsilon) \rightarrow(\log \epsilon)^{2}+K_{\alpha \beta} \log \epsilon+\frac{1}{n^{2}}\left(\log \alpha^{n}+\gamma\right)\left(\log \beta^{n}+\gamma\right)+\hat{I}_{\alpha \beta}(w ; 0)+\mathcal{O}(\epsilon)
$$




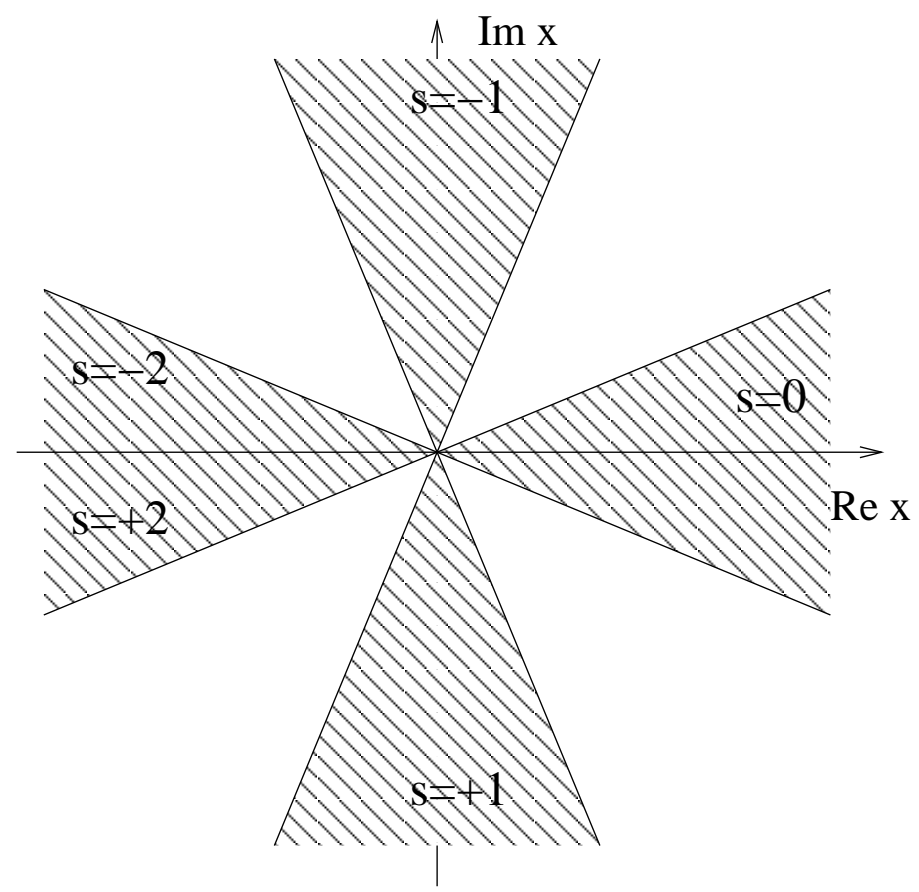

Fig 2. The angular structure in the $\alpha, \beta$ (equivalently, $x_{1}, x_{3}$ ) planes for $n=4$. In the shaded sectors the integral (4.10) converges.

In particular

$$
\frac{d}{d w} I_{\alpha, \beta}(w ; \epsilon)=\frac{d}{d w} \hat{I}_{\alpha, \beta}(w ; \epsilon)
$$

has a smooth $\epsilon \rightarrow 0$ limit.

It follows from (4.17) that if we choose linear combinations

$$
\sum c_{\alpha \beta} \hat{I}_{\alpha, \beta}(w ; 0)
$$

such that

$$
\sum c_{\alpha \beta}=0
$$

then we have a solution of the GKZ equation (4.5). Indeed, we can take the limit $\epsilon \rightarrow 0$ directly from (4.15) and we can compute the power series in $w$ explicitly. Using

$$
\int_{0}^{\infty} \frac{d t}{t} t^{k} e^{-\alpha^{n} t^{n}}=\alpha^{-k} e^{-2 \pi i \frac{k}{n} s_{\alpha}} \frac{1}{n} \Gamma\left(\frac{k}{n}\right) \quad k \geq 1
$$

we arrive at

$$
\begin{aligned}
\hat{I}_{\alpha, \beta}(w ; 0) & =\frac{1}{n^{2}} \sum_{k=1}^{\infty}(-w)^{k} \frac{(\Gamma(k / n))^{2}}{k !} e^{-2 \pi i \frac{k}{n}\left(s_{\alpha}+s_{\beta}\right)} \\
& =\frac{1}{n^{2}} \sum_{j=1}^{n} e^{-2 \pi i \frac{j}{n}\left(s_{\alpha}+s_{\beta}\right)} \hat{f}_{j-1}(w)
\end{aligned}
$$


where $\hat{f}_{m}$ was defined in (4.6). Recall that $\hat{f}_{n-1}$ does not solve the differential equation (4.5). This is in accord with (4.14).

Let us note a few important properties of the functions $\hat{I}_{\alpha, \beta}(w ; 0)$. First, it is clear from (4.22) that $\hat{I}_{\alpha, \beta}(w ; 0)$ only depends on $\alpha, \beta$ through the sector $\mathcal{S}_{s_{\alpha}} \times \mathcal{S}_{s_{\beta}}$. In fact $\hat{I}_{\alpha \beta}$ only depends on the combination $s_{\alpha \beta}:=\left(s_{\alpha}+s_{\beta}\right) \bmod n$. By definition, the function $\hat{I}_{s}$ is $\hat{I}_{\alpha \beta}$ such that $s_{\alpha \beta}=s \bmod n$. Next, note that the functions

$$
\sum c_{s} \hat{I}_{s}(w ; 0)
$$

subject to $\sum c_{s}=0$ span the space $\mathcal{V}$ of nonconstant solutions to the GKZ system. Indeed, inverting the finite Fourier transform we see that the span of $\hat{I}_{\alpha, \beta}(w ; 0)$ is the span of $\hat{f}_{0}, \ldots, \hat{f}_{n-1}$, and that $\hat{f}_{m}$ for $m<n-1$ are given by linear combinations with $\sum c_{\alpha \beta}=0$. Finally, note that $\sum c_{\alpha \beta} I_{\alpha \beta}(w ; \epsilon)$ only has a smooth limit for $\sum c_{\alpha \beta}=0$ and $\sum c_{\alpha \beta} K_{\alpha \beta}=$ 0 . In this case $\lim _{\epsilon \rightarrow 0} \sum c_{\alpha \beta} I_{\alpha \beta}=\sum c_{\alpha \beta} \hat{I}_{\alpha \beta}$.

\section{3. $L G$ symmetry}

The "LG symmetry" or "quantum symmetry" plays a fundamental role in what follows. The point $w=0$ corresponds to the orbifold point, where there is a quantum symmetry that permutes the fractional branes. From the action of this symmetry on the chiral ring generator we see that the action of the quantum symmetry is $w \rightarrow \omega w$, where $\omega=e^{2 \pi i / n}$, so a generator of the quantum symmetry takes $\hat{I}_{s} \rightarrow \hat{I}_{s+1}$, that is:

$$
\hat{I}_{s}\left(\omega^{t} w\right)=\hat{I}_{s-t}(w)
$$

Thus the set of solutions $\hat{I}_{1}-\hat{I}_{0}, \hat{I}_{2}-\hat{I}_{1}, \ldots, \hat{I}_{n-1}-\hat{I}_{n-2}, \hat{I}_{0}-\hat{I}_{n-1}$ are cyclically permuted under the quantum symmetry. The sum of these solutions is 0 . Thus, this basis is reminiscent of the the space of fractional branes orthogonal to the $D 0$ brane, and one might be tempted to identify these with the periods of fractional branes. Unfortunately, quantum monodromy is not strong enough to guarantee this and we will see that in fact a more subtle basis corresponds to the basis of fractional branes. 


\subsection{Critical points and propeller branes}

The integral representation (4.7) is useful for investigating the asymptotics of the solutions via stationary phase. In this section we set $a_{1}=a_{3}=1$ so

$$
W=x_{1}^{n}+w x_{1} x_{3}+x_{3}^{n} .
$$

Of course, solving for $W^{\prime}=0$ is the same as solving for the LG vacua. We find there are $(n-1)^{2}$ solutions. $n(n-2)$ solutions are nonzero and come in $(n-2)$ different "LandauGinzburg orbits" of the quantum $\mathbb{Z}_{n}$ symmetry $\left(x_{1}, x_{3}\right) \rightarrow\left(\omega x_{1}, \omega^{-1} x_{3}\right)$.

We should simply write:

$$
\begin{aligned}
& x_{1}=\left(-\frac{w}{n}\right)^{\frac{1}{n-2}} e^{2 \pi i \frac{\nu(n-1)}{n(n-2)}} \\
& x_{3}=\left(-\frac{w}{n}\right)^{\frac{1}{n-2}} e^{2 \pi i \frac{\nu}{n(n-2)}}
\end{aligned}
$$

with $\nu=1, \ldots, n(n-2)$. The LG $\mathbb{Z}_{n}$ symmetry is $\nu \rightarrow \nu+(n-2)$. The remaining critical point is at $\left(x_{1}, x_{3}\right)=(0,0)$.

The critical value of $W$ at (4.25) is

$$
W_{\nu}=(2-n)\left(\frac{-w}{n}\right)^{\frac{n}{n-2}} e^{2 \pi i \frac{\nu}{n-2}}
$$

Note that the different LG orbits are separated by the value of $W$ on the orbit. It is also useful to compute the Hessian:

$$
W_{\nu}^{\prime \prime}=-w\left(\begin{array}{cc}
(n-1) e^{-2 \pi i \nu / n} & -1 \\
-1 & (n-1) e^{2 \pi i \nu / n}
\end{array}\right)
$$

Note that

$$
\operatorname{det} W^{\prime \prime}=w^{2} n(n-2)
$$

does not depend on the sector. All critical points are Morse critical points.

Associated to each critical point is a vacuum state in the GLSM. Associated with each vacuum is a (topological) D-brane. To write the generalized period for the brane we define $\Gamma_{\nu}$ to be the A-brane surface defined in [12](see Section 3). In the math literature these are known as "Lefshetz thimbles" and in the context of this paper they are the propeller branes. We then define

$$
C_{\nu}(w):=\int_{\Gamma_{\nu}} e^{-W} \frac{d x_{1}}{x_{1}} \frac{d x_{3}}{x_{3}}
$$


These are well-defined for the nonzero critical points. The period $C_{0}$ for brane corresponding to $\left(x_{1}, x_{3}\right)=(0,0)$ cannot be obtained from (4.28) because the latter has a logarithmic singularity.

Although the chains $\Gamma_{\nu}$ are not $\mathbb{R}^{*} \times \mathbb{R}^{*}$ invariant the functions $C_{\nu}$ nevertheless satisfy the GKZ equation. The reason is that the chains $\Gamma_{\nu}$ approach a linear combination of the chains $\gamma_{\alpha \beta}$ at infinity, and, by Cauchy's theorem we can deform contours in a compact region without changing the integral. In fact, by examining the asymptotic behavior of the functions $C_{\nu}(w)$ at large $w$ we find that $C_{\nu}$ with $\nu=0,1, \ldots, n-2$ define a basis for the space $\mathcal{V}$, and therefore there is a locally constant matrix $M_{\nu s}$ such that

$$
C_{\nu}=\sum_{s} M_{\nu s} \hat{I}_{s}
$$

This matrix is only locally constant. It will be constant in angular sectors and will change discontinuously across angular sectors because the solutions to an equation with an irregular singular point exhibit Stokes' phenomenon.

\subsection{A basis of asymptotic solutions}

Let us now consider the asymptotic behavior of the functions in $\mathcal{V}$ at large $w$. From the saddle point formula the contribution of the critical point of type $\nu$ to $C_{\nu}$ is:

$$
\epsilon_{\nu} \frac{1}{\sqrt{n^{3}(n-2)}}\left(\frac{-w}{n}\right)^{\frac{-n}{n-2}} e^{-2 \pi i \frac{\nu}{n-2}} \exp \left[(n-2)\left(-\frac{w}{n}\right)^{\frac{n}{n-2}} e^{2 \pi i \frac{\nu}{n-2}}\right]
$$

where $\epsilon_{\nu}= \pm 1$. This is the leading term in an asymptotic expansion given by expanding the integrand of

$$
\begin{aligned}
& e^{-W_{\nu}} \int_{-\infty}^{+\infty} \frac{d t_{1} d t_{3}}{\left(x_{1}+t_{1}\right)\left(x_{3}+t_{3}\right)} e^{-\frac{1}{2}\left(\begin{array}{ll}
t_{1} & t_{3}
\end{array}\right) W^{\prime \prime}\left(\begin{array}{c}
t_{1} \\
t_{3}
\end{array}\right)} \\
& e^{-\sum_{j=3}^{n}\left(\begin{array}{c}
n \\
j
\end{array}\right) x_{1}^{n-j} t_{1}^{j}+\sum_{j=3}^{n}\left(\begin{array}{c}
n \\
j
\end{array}\right) x_{3}^{n-j} t_{3}^{j}}
\end{aligned}
$$

in powers of $t_{1}, t_{3}$ and doing the gaussian integrals. Here $\left(x_{1}, x_{3}\right)$ is the critical point we are expanding around. Note that the "interaction vertices" in the Feynman diagram expansion depend on $\nu$. The result is that (4.30) is multiplied by a power series in $w^{-n /(n-2)}$. 
These asymptotic expansions give $n-2$ asymptotic solutions to the GKZ equation, valid at large $w$. The last solution is the log solution from the asymptotic expansion at $(0,0)$. Thus, we have a basis of formal solutions:

$$
\begin{aligned}
& b_{0}=\log w-\sum_{k=1}^{\infty} \frac{\Gamma(k n)}{(k !)^{2}} w^{-k n} \\
& b_{\nu}=\frac{1}{\sqrt{n^{3}(n-2)}}\left(\frac{-w}{n}\right)^{\frac{-n}{n-2}} e^{-2 \pi i \frac{\nu}{n-2}} \exp \left[(n-2)\left(-\frac{w}{n}\right)^{\frac{n}{n-2}} e^{2 \pi i \frac{\nu}{n-2}}\right] p^{\nu} \quad \nu=1, \ldots, n-2
\end{aligned}
$$

where $p^{\nu}$ is an asymptotic series in $w^{-n /(n-2)}$.

Note that, roughly speaking, half of the series $b_{\nu}$ are "exponentially growing," and half are "exponentially decreasing." Thus, there is a filtration on $\mathcal{V}$ given by the maximal asymptotic growth as $w \rightarrow \infty$ along a fixed ray. We order the values of $\nu_{i}$ so that

$$
\operatorname{Re}\left(-\frac{w}{n}\right)^{\frac{n}{n-2}} e^{2 \pi i \frac{\nu_{1}}{n-2}}<\operatorname{Re}\left(-\frac{w}{n}\right)^{\frac{n}{n-2}} e^{2 \pi i \frac{\nu_{2}}{n-2}}<\cdots
$$

to produce the filtration

$$
\mathcal{F}_{1} \subset \mathcal{F}_{2} \subset \cdots \subset \mathcal{F}_{n-1}=\mathcal{V}
$$

Note that there are $(n-1)$ steps in the filtration because of the logarithmic solution.

Our next task is to find out how the true solutions $\sum_{s} c_{s} \hat{I}_{s}(w)$ with $\sum_{s} c_{s}=0$ fit into the filtration (4.34).

\subsection{Asymptotics of $\hat{I}_{s}$ : exponential growth}

The most natural way to investigate the asymptotics of $\hat{I}_{s}$ is to apply the saddle-point technique to the integral representation

$$
\frac{d}{d w} \hat{I}_{\alpha \beta}=-\int_{\gamma_{\alpha \beta}} e^{-W} d x_{1} d x_{3} .
$$

Applying the saddle point technique is not straightforward. Care is needed in establishing which of the saddle points (4.25) contribute to a given integral $\hat{I}_{s}$. However, if a critical point lies in the convergent sector containing the contour defining $\hat{I}_{s}$ then that critical point does contribute since no large contour deformations are required. Using that rule alone we can learn some useful facts about when $\hat{I}_{s}(w)$ has exponential growth.

Let us label the convergent sectors by $\mathcal{S}_{s}$ where $s$ is defined, modulo $n$, by (4.12).

Depending on the phase of $w$ the critical points $\left(x_{1}, x_{3}\right)_{\nu}$ in (4.25) might or might not be in a convergent sector. Note that since $x_{1}^{\nu}=x_{3}^{\nu} e^{2 \pi i \frac{\nu}{n}}$, it follows that $x_{1}, x_{3}$ either 
both lie in a convergent or in a nonconvergent sector. The condition to lie in a convergent sector is:

$$
\operatorname{Re}\left(\left(-\frac{w}{n}\right)^{\frac{n}{n-2}} e^{2 \pi i\left(\frac{\nu}{n-2}\right)}\right)>0
$$

This is precisely the criterion that $W_{\nu}<0$, and hence the same as the criterion that the contribution to an integral, if it exists, is always a growing exponential.

Let us analyze more fully when the exponentially growing critical points can contribute to an integral $I_{\alpha \beta}$. Let

$$
\left(-\frac{w}{n}\right)^{\frac{1}{n-2}} e^{2 \pi i\left(\frac{\nu}{n(n-2)}\right)}=e^{i \psi} A
$$

where $A>0$, and $-\pi<\psi<\pi$. Then from (4.36) we know there is an $s_{*}$ with

$$
-\frac{\pi}{2 n}<\psi+\frac{2 \pi}{n} s_{*}<\frac{\pi}{2 n}
$$

Then the critical point $(4.25)$ is in the sector:

$$
\left(A e^{i \psi+2 \pi i \nu / n}, A e^{i \psi}\right) \in \mathcal{S}_{s_{*}+\nu} \times \mathcal{S}_{s_{*}}
$$

Thus, for $w$ such that (4.38) holds, $\hat{I}_{\alpha \beta}$ has an exponential growth from a critical point iff $s_{\alpha \beta}=2 s_{*}+\nu \bmod n$. Moreover, the Landau-Ginzburg symmetry $\nu \rightarrow \nu+(n-2)$ relates the growth of different functions $\hat{I}_{s} \rightarrow \hat{I}_{s+1}$. To exploit this consider $\hat{I}_{0}(w)$. For $\left|\arg (-w)^{1 /(n-2)}\right|<\frac{\pi}{2}$, that is, for

$$
-\frac{\pi}{2}+\frac{\pi}{n}<\arg (-w)<\frac{\pi}{2}-\frac{\pi}{n}
$$

the critical point $\nu=n(n-2) \cong 0$ contributes to the integral. Therefore, $\hat{I}_{0}$ has exponential growth in this sector. Now using $\hat{I}_{s}(w)=\hat{I}_{0}\left(\omega^{-s} w\right)$ we can make similar statements about the other sectors.

As we shall see in the next section, in the sector complementary to (4.40) $\hat{I}_{0}$ has in fact at most logarithmic growth. In overlapping sectors of the type (4.40) we can form linear combinations of the $\hat{I}_{s}$ to produce functions with exponential growth slower than the leading one.

\section{Examples}

1. $n=3$. The functions $\hat{I}_{s}$ have exponential growth like $\mathcal{E}=-\sqrt{27} w^{-3} \exp \left((-w / 3)^{3}\right)$ for:

$$
\begin{array}{ll}
\hat{I}_{0}: & -\frac{\pi}{6}<\arg (-w)<\frac{\pi}{6} \\
\hat{I}_{1}: & -\frac{5 \pi}{6}<\arg (-w)<-\frac{3 \pi}{6} \\
\hat{I}_{2}: & \frac{3 \pi}{6}<\arg (-w)<\frac{5 \pi}{6}
\end{array}
$$


These are the shaded regions in Fig. 3 below.

2. $n=4$. We have exponential growth for

$$
\begin{array}{ll}
\hat{I}_{0}: & -\frac{\pi}{4}<\arg (-w)<\frac{\pi}{4} \\
\hat{I}_{1}: & \frac{\pi}{4}<\arg (-w)<\frac{3 \pi}{4} \\
\hat{I}_{2}: & \frac{3 \pi}{4}<\arg (-w)<\frac{5 \pi}{4} \\
\hat{I}_{3}: & \frac{-3 \pi}{4}<\arg (-w)<\frac{-\pi}{4}
\end{array}
$$

See Fig. 4.

\subsection{Asymptotics of $\hat{I}_{s}$ : Coefficient of the logarithm}

In this section we introduce a different integral representation for the functions $\hat{I}_{s}$ which, while only valid in part of the complex $w$ plane, is very useful for extracting asymptotic behavior for $|w| \rightarrow \infty$. We apply a method described in [18]. 6

In what follows $\arg (z)$ always means the principal branch of the logarithm, so it is defined for $z \in \mathbb{C}-\mathbb{R}^{-}$and $|\arg (z)|<\pi$. We begin with

$$
e^{-z}=\frac{1}{2 \pi i} \int_{\mathcal{C}} \Gamma(u) z^{-u} d u \quad|\arg (z)|<\pi / 2
$$

where $\mathcal{C}$ is the contour $u=\epsilon+i y, y \in \mathbb{R}, \epsilon>0$. Note that

$$
|\Gamma(x+i y)| \sim \sqrt{2 \pi}|y|^{-\frac{1}{2}+x} e^{-\frac{\pi}{2}|y|}
$$

for $y \rightarrow \pm \infty$ at any fixed $x$. Thus the integrand converges absolutely for $|\arg (z)|<\pi / 2$. To prove (4.43) note that we can close the contour in the left half plane.

We apply this to $e^{-\alpha \beta w t_{1} t_{3}}$ in the integral representation for $\hat{I}$ which follows from (4.10). Thus we must require

$$
|\arg (\alpha \beta w)|<\frac{\pi}{2}
$$

and for such values of $\alpha, \beta, w$ we define the integer $N(\alpha, \beta, w)$ by

$$
-\frac{\pi}{2}<\arg (\alpha)+\arg (\beta)+\arg (w)+2 \pi N(\alpha, \beta, w)<\frac{\pi}{2}
$$

6 This reference examines asymptotics of the integrals relevant to the more general set of $\mathbb{C}^{2} / \mathbb{Z}_{n(p)}$ models and might be useful in further extensions of the present paper. 
The integrals are absolutely convergent and we can exchange them and do the $t_{1}, t_{3}$ integral. Using the generalization of (4.21)

$$
\int_{0}^{\infty} \frac{d t}{t} t^{-s} e^{-\alpha^{n} t^{n}}=\alpha^{s-1} e^{\frac{2 \pi i s_{\alpha}}{n}(s-1)} \frac{1}{n} \Gamma\left(\frac{1-s}{n}\right) \quad \operatorname{Re}(s)<1
$$

we find

$\frac{d}{d w} \hat{I}_{\alpha \beta}(w ; 0)=-e^{-2 \pi i s_{\alpha \beta} / n} \frac{1}{2 \pi i n^{2}} \int_{\mathcal{C}} \Gamma(u)\left(\Gamma\left(\frac{1-u}{n}\right)\right)^{2} w^{-u} \exp \left[2 \pi i u\left(\frac{s_{\alpha \beta}}{n}-N(\alpha, \beta, w)\right)\right] d u$

This is valid in the region (4.45). It is a good exercise to use (4.44) to check that (4.48) is an absolutely convergent integral in this range. Indeed, this condition guarantees absolute convergence of the integral in (4.48) along any contour of the form $x+i y$ for fixed $x$ with $y \in \mathbb{R}$.

Now, using the property that $I_{\alpha \beta}$ only depends on the wedge in which $\alpha, \beta$ live we can map out the range of validity for the integral representation (4.48). It follows that, for $\hat{I}_{0}$, the domain of validity of $(4.48)$ is the region

$$
-\frac{\pi}{2}-\frac{\pi}{n}<\arg (w)<\frac{\pi}{2}+\frac{\pi}{n}
$$

Note that this is perfectly complementary to the region (4.40). Using the LG symmetry we find that the formula (4.48) for $\hat{I}_{s}$ holds in the range

$$
-\frac{\pi}{2}+\frac{\pi}{n}(2 s-1)<\arg w<\frac{\pi}{2}+\frac{\pi}{n}(2 s+1)
$$

If $n$ is sufficiently larger then $s$ then $N=0$ throughout (4.50).

The integral (4.48) is useful because it allows us to obtain asymptotic expansions for $\hat{I}_{s}$ in the region (4.50). While we cannot close the $u$-contour integral in the left half plane, we can displace the contour to the right, thanks to (4.44). In the process we pick up poles from $u=1+k n, k=0,1,2, \ldots$. In this way we arrive at the asymptotic expansion

$$
\frac{d}{d w} \hat{I}_{\alpha \beta}(w ; 0) \sim 2 \pi i\left(\frac{s_{\alpha \beta}}{n}-N(\alpha, \beta, w)\right) \sum_{k=0}^{\infty} \frac{\Gamma(1+k n)}{(k !)^{2}} w^{-1-k n}+\sum_{k=0}^{\infty} D_{k}(n) w^{-1-k n}
$$

where the $D_{k}(n)$ are functions of $k, n$ but are independent of $w$ and, crucially, are independent of $\alpha, \beta$ and hence cancel out when one forms combinations $\sum c_{\alpha \beta} \hat{I}_{\alpha \beta}$ such that 
$\sum c_{\alpha \beta}=0$. Integrating this formula we have the asymptotic expansion

$$
\begin{aligned}
\hat{I}_{\alpha \beta}(w ; 0) & \sim 2 \pi i\left(\frac{s_{\alpha \beta}}{n}-N(\alpha, \beta, w)\right)\left(\log w-\sum_{k \geq 1} \frac{\Gamma(k n)}{(k !)^{2}} w^{-k n}\right)+\mathcal{U} \\
\mathcal{U} & :=-\frac{1}{2}(\log w)^{2}-\frac{(n-2)}{n} \gamma \log w+c+\sum_{k \geq 1} \frac{\Gamma(k n)}{(k !)^{2}}\left(-k n w^{-k n} \log w+h_{k} w^{-k n}\right) \\
h_{k} & :=-1+\frac{2}{n}\left(1+\frac{1}{2}+\cdots+\frac{1}{k}-\gamma\right)-\Psi(1+k n)
\end{aligned}
$$

where $c$ is a constant. The important thing in this formula is that $\mathcal{U}$ is independent of $\alpha, \beta$ (and hence of $s$ ). Note that the $s$-dependent term is nicely consistent with the logarithmic dependence on $w$ in the expansion $\mathcal{U}$, and the LG symmetry. The formula is valid in the LG images of (4.49). The asymptotics perfectly complement the region (4.40) where the leading exponential dominates.

One important conclusion we can draw from (4.52) is that in those regions where (4.45) is simultaneously valid for all terms with $c_{\alpha \beta} \neq 0$ and such that $\sum c_{\alpha \beta}\left(\frac{s_{\alpha \beta}}{n}-N(\alpha, \beta, w)\right)=$ 0 then $\sum c_{\alpha \beta} \hat{I}_{\alpha \beta}$ will be an exponentially decaying solution.

The discussion in this section falls short of giving a complete description of the filtration (4.34) in all angular sectors for general $n$ because we have not explained how to form linear combinations with prescribed sub-exponential growth. One can apply the saddle point technique to the integral (4.48) for linear combinations, such as $\hat{I}_{s}+\hat{I}_{-s}-2 \hat{I}_{0}$ for which the pole terms cancel. One finds that $\hat{I}_{s}$ contributes an exponential behavior like

$$
\begin{cases}\exp \left[-(n-2)\left(\frac{w}{n}\right)^{\frac{n}{n-2}} e^{-\frac{2 \pi i}{n-2}(s+1)}\right] & \operatorname{Im} w>0 \\ \exp \left[-(n-2)\left(\frac{w}{n}\right)^{\frac{n}{n-2}} e^{-\frac{2 \pi i}{n-2}(s-1)}\right] & \operatorname{Im} w<0\end{cases}
$$

in the intersection of the regions (4.50). (We assume $n \gg|s|$ at this point.) The term with the least rapid decay will then dominate. Careful application of this rule might suffice to determine the full filtration (4.34) but we have not carried this out. There is also a Mellin-Barnes representation of the functions $\hat{f}_{m}$ but the saddle point technique applied to this representation proves inconclusive. Nevertheless, the results we have presented here do suffice to give a rather complete picture of the filtration for $n=3$ and $n=4$, as we describe in the next subsection. 


\subsection{Examples: $n=3$ and $n=4$}

Let us show how the above general results can give a complete picture of the filtration for the cases $n=3$ and $n=4$. Let

$$
\mathcal{E}=-\sqrt{27} w^{-3} \exp \left(-\frac{w}{3}\right)^{3}
$$

Then we have

$$
\begin{gathered}
\hat{I}_{0} \sim \begin{cases}\mathcal{U} & -\frac{5 \pi}{6}<\arg w<\frac{5 \pi}{6} \\
\mathcal{E} & -\frac{\pi}{6}<\arg -w<\frac{\pi}{6}\end{cases} \\
\hat{I}_{1} \sim \begin{cases}\mathcal{U}+\frac{2 \pi i}{3} \log w+\cdots & -\frac{\pi}{6}<\arg w<\pi \\
\mathcal{U}-\frac{4 \pi i}{3} \log w+\cdots & -\pi<\arg w<-\frac{\pi}{2} \\
\mathcal{E} & -\frac{\pi}{2}<\arg -w<-\frac{\pi}{6}\end{cases} \\
\hat{I}_{-1} \sim \begin{cases}\mathcal{U}+\frac{4 \pi i}{3} \log w+\cdots & \frac{\pi}{2}<\arg w<\pi \\
\mathcal{U}-\frac{2 \pi i}{3} \log w+\cdots & -\pi<\arg w<\frac{\pi}{6} \\
\mathcal{E} & \frac{\pi}{6}<\arg -w<\frac{\pi}{2}\end{cases}
\end{gathered}
$$

Now let us describe the corresponding filtrations. There are only two steps in (4.34). In the "convergent sectors" $\operatorname{Re} w^{3}<0$ the exponential solution, which is asymptotic to $\mathcal{E}$, is growing and we have $\mathcal{F}_{0} \subset \mathcal{F}_{+}$where $\mathcal{F}_{0}$ is the 1 -dimensional space spanned by the log solution. In the sectors with $\operatorname{Re} w^{3}>0, \mathcal{E}$ is decaying and we have the filtration $\mathcal{F}_{-} \subset \mathcal{F}_{0}$, where $\mathcal{F}_{-}$is the one-dimensional space spanned by the exponentially decaying solution $\mathcal{E}$.

The first step in the filtration is given by

$$
\mathcal{F}_{0}=\operatorname{Span} \begin{cases}\hat{I}_{0}-\hat{I}_{-1} \sim \frac{2 \pi i}{3} \log w+\cdots & -\frac{3 \pi}{6}<\arg w<-\frac{\pi}{6} \\ \hat{I}_{1}-\hat{I}_{-1} \sim-\frac{2 \pi i}{3} \log w+\cdots & -\frac{\pi}{6}<\arg (-w)<\frac{\pi}{6} \\ \hat{I}_{0}-\hat{I}_{1} \sim-\frac{2 \pi i}{3} \log w+\cdots & \frac{\pi}{6}<\arg w<\frac{3 \pi}{6}\end{cases}
$$

for the sectors in which $\mathcal{E}$ is exponentially growing. Note the three lines of (4.58) are related by LG symmetry. Similarly the first step in the filtration is given by

$$
\mathcal{F}_{-}=\operatorname{Span} \begin{cases}2 \hat{I}_{0}-\hat{I}_{-1}-\hat{I}_{+1} \sim \text { cnst. } & -\frac{\pi}{6}<\arg w<\frac{\pi}{6} \\ 2 \hat{I}_{1}-\hat{I}_{-1}-\hat{I}_{0} \sim \text { cnst. } & \frac{3 \pi}{6}<\arg w<\frac{5 \pi}{6} \\ 2 \hat{I}_{-1}-\hat{I}_{1}-\hat{I}_{0} \sim \text { cnst.E } & -\frac{5 \pi}{6}<\arg w<-\frac{3 \pi}{6}\end{cases}
$$

for the sectors in which $\mathcal{E}$ is exponentially decreasing.

Similarly, for $n=4$ we find:

$$
\hat{I}_{0} \sim \begin{cases}\mathcal{U} & -\frac{3 \pi}{4}<\arg w<\frac{3 \pi}{4} \\ \mathcal{E}_{0} & -\frac{\pi}{4}<\arg (-w)<\frac{\pi}{4}\end{cases}
$$


Here $\mathcal{E}_{0} \sim \sqrt{2} w^{-2} \exp \left[w^{2} / 8\right]$ is the growing exponential corresponding to the critical point with $\nu=0$ (or its LG images).

$$
\hat{I}_{1} \sim \begin{cases}\mathcal{U}+\frac{2 \pi i}{4} \log w+\cdots & -\frac{\pi}{4}<\arg w<\pi \\ \mathcal{U}-\frac{3 \pi i}{4} \log w+\cdots & -\pi<\arg w<-\frac{3 \pi}{4} \\ \mathcal{E}_{1} & -\frac{3 \pi}{4}<\arg w<-\frac{2 \pi}{4}\end{cases}
$$

Here $\mathcal{E}_{1} \sim \sqrt{2} w^{-2} \exp \left[-w^{2} / 8\right]$ is the growing exponential corresponding to the critical point with $\nu=1$ (or its LG images). Similarly

$$
\begin{gathered}
\hat{I}_{-1} \sim \begin{cases}\mathcal{U}-\frac{2 \pi i}{4} \log w+\cdots & -\pi<\arg w<\frac{\pi}{4} \\
\mathcal{U}+\frac{3 \pi i}{4} \log w+\cdots & \frac{3 \pi}{4}<\arg w<\pi \\
\mathcal{E}_{1} & \frac{\pi}{4}<\arg w<\frac{3 \pi}{4}\end{cases} \\
\hat{I}_{2} \sim \begin{cases}\mathcal{U}+i \pi \log w+\cdots & \frac{\pi}{4}<\arg w<\pi \\
\mathcal{U}-i \pi \log w+\cdots & -\pi<\arg w<-\frac{\pi}{4} \\
\mathcal{E}_{0} & -\frac{\pi}{4}<\arg w<\frac{\pi}{4}\end{cases}
\end{gathered}
$$

Using these formulae we can specify bases for the 3-step filtration $\mathcal{F}_{-} \subset \mathcal{F}_{0} \subset \mathcal{F}_{+}$, where $\mathcal{F}_{-}$has at most exponential decay, and $\mathcal{F}_{0}$ has at most logarithmic growth. In the sectors $|\arg w|<\frac{\pi}{4}$ we find that $\mathcal{F}_{-}$is generated by $2 \hat{I}_{0}-\hat{I}_{1}-\hat{I}_{-1} \sim$ cnst. $\mathcal{E}_{1}$, while $\mathcal{F}_{0}$ is spanned by $\hat{I}_{0}-\hat{I}_{1}$ and $\hat{I}_{0}-\hat{I}_{-1}$. The filtrations in the other sectors are given by applying the LG symmetry.

\subsection{Stokes matrices}

The differential equation (4.5) can be written as a first order $n \times n$ matrix equation of the form:

$$
\left(\frac{d}{d w}-A(w)\right) \Psi=0
$$

where

$$
A(w)=\sum_{i=1}^{n-1} e_{i, i+1}+\frac{(-1)^{n}}{n^{2}} w e_{n, 2}+\frac{(-1)^{n}}{n^{2}} w^{2} e_{n, 3}
$$

and $e_{i, j}$ are matrix units. We regard this as an equation for an $n \times n$ invertible matrix $\Psi$. The physical interpretation of $\Psi$ is that it is the matrix of 1-point correlators of the elements of the chiral ring $\left(\mathbb{C}\left[x_{1}, x_{3}\right] /\left(x_{i} \partial_{i} W\right)\right)^{\mathbb{Z}_{n}}$ :

$$
\Psi_{i j}=\int_{\gamma_{i}}\left(x_{1} x_{3}\right)^{j} e^{-W} \frac{d x_{1}}{x_{1}} \frac{d x_{3}}{x_{3}}
$$

At infinity there is a formal asymptotic solution. True $n \times n$ matrix solutions asymptotic to the fixed formal solution can only be defined in angular sectors. For sectors of sufficiently 
wide angle the true solution is unique. On overlapping sectors two such solutions will be related by right multiplication by a constant matrix known as a Stokes matrix. For further details see, for examples, [19][20].

The results of the previous sections allow one to determine the Stokes matrices for $n=3,4$. We work directly with a basis for $\mathcal{V}$, rather then $\Psi$. For $n=3$ we define a vector of formal solutions

$$
\left(\begin{array}{c}
\kappa_{0} b_{0} \\
\kappa_{1} b_{1}
\end{array}\right)
$$

where $\kappa_{0}, \kappa_{1}$ are appropriate constants and $b_{0}, b_{1}$ are defined in (4.32). We now introduce six sectors: $\mathcal{S}_{j}:=\left\{w \mid \frac{\pi j}{3}-\frac{\pi}{2}<\arg w<\frac{\pi j}{3}+\frac{\pi}{6}\right\}, 1 \leq j \leq 6$. In each of these sectors there is a unique basis of solutions asymptotic to (4.67). These are:

$$
\begin{array}{lll}
\mathcal{S}_{1}: & \psi_{1}=\left(\begin{array}{c}
\hat{I}_{0}-\hat{I}_{1} \\
2 \hat{I}_{0}-\hat{I}_{1}-\hat{I}_{-1}
\end{array}\right) \\
\mathcal{S}_{2}: & \psi_{2}=\left(\begin{array}{c}
\hat{I}_{0}-\hat{I}_{1} \\
2 \hat{I}_{1}-\hat{I}_{0}-\hat{I}_{-1}
\end{array}\right) \\
\mathcal{S}_{3}: & \psi_{3}=\left(\begin{array}{c}
\hat{I}_{1}-\hat{I}_{-1} \\
2 \hat{I}_{1}-\hat{I}_{0}-\hat{I}_{-1}
\end{array}\right)
\end{array}
$$

The other sectors are obtained by LG symmetry. Then we have Stokes matrices:

$$
\begin{aligned}
\mathcal{S}_{1} \cap \mathcal{S}_{2}: & \psi_{1}=\left(\begin{array}{cc}
1 & 0 \\
3 & 1
\end{array}\right) \psi_{2} \\
\mathcal{S}_{2} \cap \mathcal{S}_{3}: & \psi_{2}=\left(\begin{array}{cc}
1 & -1 \\
0 & 1
\end{array}\right) \psi_{3}
\end{aligned}
$$

and the remaining sectors are obtained by applying LG symmetry. Similarly, one can compute the Stokes matrices for $n=4$.

\subsection{Summary}

The generalized periods are solutions of the GKZ equation (4.5). A basis of solutions can be written in the integral representation (4.7) (4.35) for appropriate linear combinations of regulated "straightline contours" (4.9). These linear combinations should be thought of as elements of the homology group $H_{n}\left(\mathbb{C}^{2}, B\right)$ where $B$ is the region at infinity where $\operatorname{Re} W \rightarrow+\infty$. By combining the "Landau-Ginzburg symmetry" (4.24) with saddle point techniques ( equations (4.25)(4.26) 4.30) and the integral representation (4.48) we are able to find the angular-sector-dependent asymptotic behavior of the generalized periods, which exhibit Stokes' phenomenon. We gave a detailed analysis for the cases of $n=3,4$ in (4.54)-(4.63) and showed how to compute the Stokes' matrices in (4.69). 


\section{Propeller branes vs. fractional branes: analysis for small $n$}

In this section we analyze $\mathbb{C}^{2} / \mathbb{Z}_{n(p=1)}$ orbifolds for $n=3,4$. Let us first summarize our findings. We look at the overlap of the Ramond ground state with the branes in the theory deformed by the addition of a tachyon vertex operator. This quantity is the direct analog of the central charge in the spacetime supersymmetric theories and we call it the "generalized central charge" or "generalized period." Earlier in the paper we explained that this quantity satisfies the GKZ equation. A convenient basis of non-constant solutions is provided by combinations of $\hat{I}_{s}$ which satisfy $(4.23)$.

As $x_{1}, x_{3} \rightarrow \infty$, the A-brane surfaces defined in Section 3 asymptote to quarterplanes (4.9) used in the definition of $I_{\alpha, \beta}$ in (4.10). More precisely, we find that

$$
\left\langle c_{\nu} \mid 1\right\rangle=\mathcal{C}_{1}+\mathcal{C}_{2}\left(\hat{I}_{s+1}+\hat{I}_{s-1}-2 \hat{I}_{s}\right)
$$

with the relation between $\nu$ and $s$ to be determined later. In eq. (5.1) $\mathcal{C}_{1}$ and $\mathcal{C}_{2}$ are $w$-independent constants. We do not know the behavior of the contour $\Gamma_{\nu}$ in the compact region. Because of the pole $1 / x_{1} x_{3}$ in the measure we must allow for a constant term $C_{1}$ which cannot be determined by our techniques]. In the following, we will adopt the notation

$$
\left\langle c_{\nu} \mid 1\right\rangle \sim \hat{I}_{s+1}+\hat{I}_{s-1}-2 \hat{I}_{s}
$$

which is meant to be equivalent to (5.1). The evidence for (5.2) comes from numerically solving the differential equations that define the profile of the A-brane. [We also observe that $(5.2)$ is consistent with the saddle-point evaluation of the integral (3.3).]

The theory at the orbifold point contains a set of fractional branes whose boundary states are described in appendix A. In the following we will provide evidence that the coulomb branch branes are equivalent to the fractional branes, up to a permutation. In fact, we propose

$$
\left\langle e_{a} \mid 1\right\rangle=\mathcal{C}_{2}\left(\hat{I}_{a+1}+\hat{I}_{a-1}-2 \hat{I}_{a}\right)+\frac{1}{n}
$$

(From the exact boundary state description we happen to know the value of the constant term for this overlap; it is equal to $1 / n$.) The evidence for (5.3) comes from the map between the higgs branch brane and the fractional branes, which is determined by the intersection matrix. In fact, for $n=3$ the map also implies that the coulomb branch brane

7 Recall that the intersection matrix is also insensitive to the addition/subtraction of D0 branes, which corresponds to adding/subtracting an integer from (5.1). 
is equal to the fractional brane up to permutations. Here is a consistency check for (5.3). Consider turning on the tachyon vertex operator $w$. The worldsheet action of the orbifold theory is modified by

$$
\delta S \sim w \int d z d \bar{z} \int d^{2} \theta X_{\frac{1}{n}} .
$$

The disk one-point function of the unit operator to first nontrivial order in $w$ is

$$
\left\langle e_{a} \mid 1\right\rangle \sim w \exp \left(-\frac{2 \pi i a}{n}\right)+\mathcal{O}\left(w^{2}\right)
$$

where we used the boundary state expression for the fractional brane to determine the onepoint function, and we have dropped $n$-dependent normalization constants. Substituting the small $w$ expansion (4.22) into (5.3) gives precisely (5.5).

\section{1. $n=3$}

We first consider the case of $n=3, p=1$. In fact, the orbifold group is $Z_{N=2 n}=Z_{6}$ due to the action on fermions; only the type 0 (not the type II) theory can be defined. There are three choices for the intersection matrix for fractional branes, given by (2.34) and by matrices obtained from (2.34) by permutation of columns.

(1) The complete, unreduced intersection matrix, obtained by a permutation of columns from (2.34) is

$$
\mathcal{I}=\left(\begin{array}{ccc}
-2 & 1 & 1 \\
1 & -2 & 1 \\
1 & 1 & -2
\end{array}\right)
$$

The reduced matrix, with the D0 brane factored out, corresponds to the continued fraction determined by $n /(n-p) \rightarrow[2,2]$.

$$
\tilde{\mathcal{I}}=\left(\begin{array}{cc}
-2 & 1 \\
1 & -2
\end{array}\right)
$$

The corresponding Hirzebruch-Jung space is an ALE space. It is obtained from the orbifold by turning on the generators of the $(c, c)$ ring. This is essentially equivalent to the spacetime supersymmetric case. There is no coulomb branch and the map between the fractional branes and the higgs branch branes is known via the McKay correspondence.

(2) The unreduced matrix is obtained by a permutation of columns in (5.6). The reduced intersection form is determined by the continued fraction determined by $n / p \rightarrow[3]$.

$$
\tilde{\mathcal{I}}=\left(\begin{array}{cc}
1 & -2 \\
1 & 1
\end{array}\right)
$$


According to (2.30), the higgs and the coulomb branch branes are given by

$$
h=e_{s}-e_{s+1}, \quad c=e_{s+1}
$$

where the index $s$ is not determined at this stage. In fact, as we will see below, all permutations will be realized, depending on the argument of $w$.

(3) Similar to the previous case, the continued fraction is $n / p \rightarrow[3]$ and

$$
\tilde{\mathcal{I}}=\left(\begin{array}{cc}
1 & 1 \\
-2 & 1
\end{array}\right)
$$

and therefore

$$
h=e_{s}+2 e_{s+1}, \quad c=e_{s+1}
$$

To proceed further, it will be convenient to have a map of the $w$-plane divided into angular sectors. This is shown in Fig. 3.

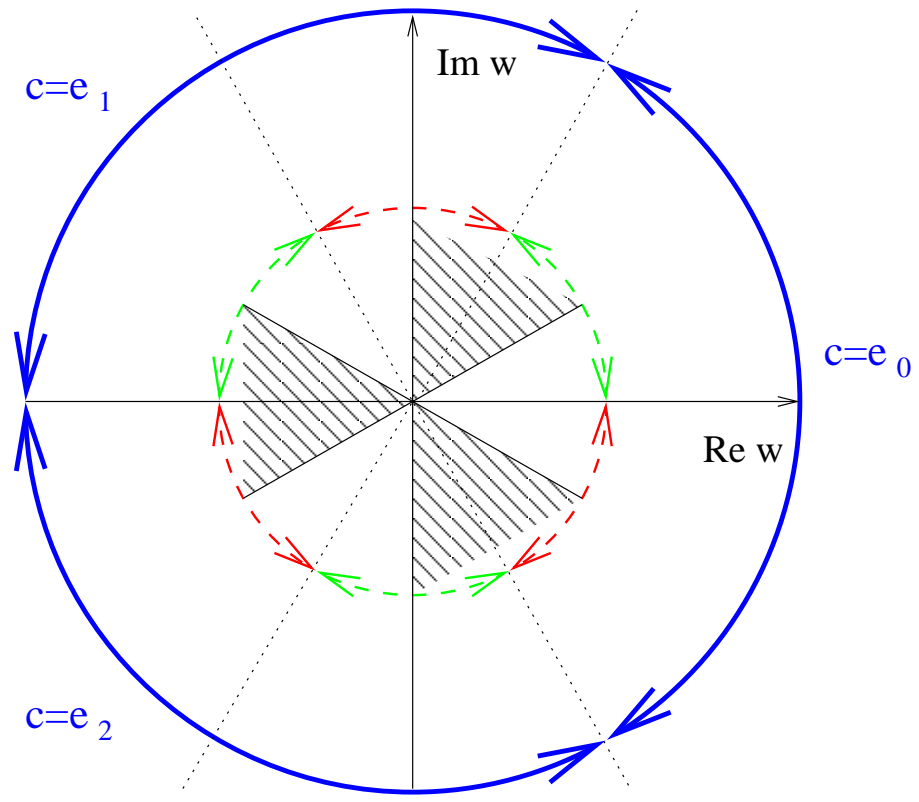

Fig 3. The angular structure in the $w$ plane. $w$ in the shaded sectors gives rise to a critical point with a negative value of ReW. Big blue arrows mark sectors which differ by permutations of fractional branes. Dashed red and green arrows mark sectors which differ by the type of higgs branch brane 
According to (4.25), modulo the LG symmetry, there is a single critical point of the superpotential $W$. Its coordinates, and the value of $W$ are

$$
x_{1}=x_{3}=-\frac{w}{3}, \quad W_{*}=\left(\frac{w}{3}\right)^{3}
$$

Hence there are three angular sectors in the $w$-plane where $W_{*}$ is negative and the integral (3.3) can pick up an exponentially growing contribution from the critical point. This happens when $\left(x_{1}, x_{3}\right)$ given by (5.12) fall into the regions (see (4.42) above)

$$
\begin{array}{rlrl}
\frac{5 \pi}{6} & \leq \arg (w) \leq \frac{7 \pi}{6}, & & s=0 \\
\frac{3 \pi}{2} & \leq \arg (w) \leq \frac{11 \pi}{6}, & & s=1 \\
\frac{\pi}{6} & \leq \arg (w) \leq \frac{\pi}{2}, & s & s=-1
\end{array}
$$

These are the shaded sectors in Fig. 3. The value of $s$ in (5.13) specifies which $\hat{I}_{s}$ receives an exponentially growing contribution, in accord with the rule (4.39). In these sectors, the nonconstant solutions of the GKZ equation are spanned by the two functions with the following leading asymptotics:

$$
\begin{array}{rlrl}
\frac{5 \pi}{6} & \leq \arg (w) \leq \frac{7 \pi}{6}: & \hat{I}_{-1}-\hat{I}_{1} \sim \frac{2 \pi i}{3} \log w, & \hat{I}_{0}-I_{-1} \sim \exp \left(-W_{*}\right) \\
\frac{3 \pi}{2} \leq \arg (w) \leq \frac{11 \pi}{6}: & \hat{I}_{0}-\hat{I}_{-1} \sim \frac{2 \pi i}{3} \log w, & \hat{I}_{1}-I_{0} \sim \exp \left(-W_{*}\right) \\
\frac{\pi}{6} \leq \arg (w) \leq \frac{\pi}{2}: & \hat{I}_{1}-\hat{I}_{0} \sim \frac{2 \pi i}{3} \log w, & \hat{I}_{-1}-I_{1} \sim \exp \left(-W_{*}\right)
\end{array}
$$

where $W_{*}$ is given by (5.12) and is a growing exponential. The precise form of the exponential growth is given in (4.54), but we use $e^{-W_{*}}$ as a shorthand for $\mathcal{E}$. Note that the three sectors are different by a simple permutation of indices of $\hat{I}$ 's. We will see below that this is a general phenomenon which is rooted in the permutation symmetry of the fractional branes.

Consider now three sectors where $R e W_{*}$ is positive, and the contribution from the nonzero critical point is a decaying exponential. These are the unshaded sectors in Fig. 3. The leading contribution now comes from the critical point at $\left(x_{1}, x_{3}\right)=(0,0)$ and is logarithmic.

$$
\begin{array}{rlrlrl}
-\frac{\pi}{6} & \leq \arg (w) \leq \frac{\pi}{6}: & \hat{I}_{1}-\hat{I}_{0} \sim \frac{2 \pi i}{3} \log w, & \langle c \mid 1\rangle \sim \hat{I}_{1}+\hat{I}_{-1}-2 \hat{I}_{0} \sim \exp \left(-W_{*}\right) \\
\frac{\pi}{2} \leq \arg (w) \leq \frac{5 \pi}{6}: & \hat{I}_{-1}-\hat{I}_{1} \sim \frac{2 \pi i}{3} \log w, & \langle c \mid 1\rangle \sim \hat{I}_{-1}+\hat{I}_{0}-2 \hat{I}_{1} \sim \exp \left(-W_{*}\right) \\
\frac{7 \pi}{6} \leq \arg (w) \leq \frac{3 \pi}{2}: & \hat{I}_{0}-\hat{I}_{-1} \sim \frac{2 \pi i}{3} \log w, & \langle c \mid 1\rangle \sim \hat{I}_{0}+\hat{I}_{1}-2 \hat{I}_{-1} \sim \exp \left(-W_{*}\right)
\end{array}
$$


In eq. (5.15) we identified the exponentially decaying solution with the generalized central charge of the coulomb branch brane. Indeed, we expect the latter to receive an exponentially decaying contribution from the critical point of $W$. Now both (5.9) and (5.11) state that the coulomb branch brane is the same as the fractional brane, up to a permutation. But we can fix the freedom with the help of (5.5). This leads to (5.3).

The picture that we infer from (5.15) is therefore the following. In the (undashed) sector $-\frac{\pi}{6} \leq \arg (w) \leq \frac{\pi}{6}, c=e_{0}$. Rotating $\arg (w)$ by $2 \pi / 3$ enforces the permutation of fractional branes. E.g. in the sector $\frac{\pi}{2} \leq \arg (w) \leq \frac{5 \pi}{6}, c=e_{1}$ etc. What we do not yet know is how the transition between $c=e_{0}$ and $c=e_{1}$ (and more generally, between $c=e_{s}$ and $c=e_{s+1}$ ) happens. A natural scenario would be the following. The $w$ plane is divided into 3 angular regions, marked by the blue arrows in Fig. 3 . In the region $-\pi / 3 \leq \arg (w) \leq \pi / 3, c=e_{0}$, and $w \rightarrow \exp (2 \pi i / 3) w$ enforces the permutation of the fractional branes $e_{s} \rightarrow e_{s+1}$. Unfortunately we cannot see this directly from the asymptotics of $\hat{I}^{\prime}$ 's. The reason is that both $e_{s}$ and $e_{s+1}$ have the same exponentially growing asymptotics in the region where the transition happens. However there is a way to verify the picture proposed above. As explained before, the shape of the coulomb branch brane can be inferred from the solution of the soliton equations. The corresponding solutions emanate from the critical point of $W$ and run to infinity along the quarterplanes $\gamma_{\alpha \beta}$ used to define the $\hat{I}_{\alpha \beta}$ 's (there are $n=3$ inequivalent choices of them). Solving the equations near the critical point and far away, in the asymptotic region, is easy. The more difficult question is matching the solutions in the two regions, i.e. understanding which direction in the $w$ plane is chosen by a certain soliton trajectory, and how this choice depends on $\arg (w)$. In appendix B we analyze this question numerically. Our analysis confirms the picture described above. That is, there are three angular sectors in the $w$ plane, and the asymptotics of the A-brane surface emanating from the nonzero critical point (i.e. coulomb branch brane) jump as $w$ crosses the lines of $\arg (w)=\pi / 3, \pi,-\pi / 3$. Moreover, the asymptotics are consistent with (5.15).

So far we have determined the behavior of the generalized central charge for the coulomb branch brane as a function of $w$. The theory contains a single higgs branch brane, which wraps the exceptional $\mathbb{P}^{1}$ of $\mathcal{O}(-3) \rightarrow \mathbb{P}^{1}$. Unfortunately we cannot analyze the soliton equations for this brane, since the LG model is unreliable near $\left(x_{1}, x_{3}\right)=(0,0)$ which would serve as a critical point. Nevertheless, we can use (5.9) and (5.11) together with (5.3) to compute the generalized central charge. Let us specialize the discussion to the angular sector $-\pi / 6 \leq \arg (w) \leq \pi / 6$; the other two sectors differ by permutation. 
In this sector $c=e_{0}$, therefore the two possibilities for the higgs branch brane consistent with the intersection matrix are $h^{(1)}=e_{2}-e_{0}$ and $h^{(2)}=e_{2}+2 e_{0}$. (Again, one must keep in mind that the expression for the the higgs and coulomb branch branes are obtained modulo the addition/subtraction of D0 branes) Using (5.3) we obtain

$$
\left\langle h^{(1)} \mid 1\right\rangle \sim 3\left(\hat{I}_{0}-\hat{I}_{-1}\right), \quad\left\langle h^{(2)} \mid 1\right\rangle \sim 3\left(\hat{I}_{1}-\hat{I}_{0}\right)
$$

We can identify the logarithmic solution with the higgs branch brane for the shaded sectors in the $w$ plane. This is where it is defined uniquely (in the unshaded sectors, one can add an exponentially decaying solution without changing the leading asymptotics). Comparing with (5.14) we observe that for $-\pi / 3 \leq \arg (w) \leq-\pi / 6, h^{(1)}=e_{2}-e_{0}$, while for $\pi / 6 \leq$ $\arg (w) \leq \pi / 3, h^{(2)}=e_{2}+2 e_{0}$. The natural line of transition between the two happens at $\arg (w)=0$. (This would be a direct analog of the transition between $c=e_{s}$ and $c=e_{s+1}$ ). We cannot rigorously prove this point and leave further justification to the future 8 .

Let us summarize. There are $n=3$ "big" angular sectors. In the sector $-\pi / 3 \leq$ $\arg (w) \leq \pi / 3$, the coulomb branch brane $c=e_{0}$. Multiplication by $\exp (2 \pi i / 3)$ causes cyclic permutations of the fractional branes $e_{0} \rightarrow e_{1}$ etc. In addition, each big sector is divided into two smaller ones. For example, in the angular sector $-\pi / 3 \leq \arg (w) \leq \pi / 3$ there are two possible higgs branch branes:

$$
\begin{aligned}
& h^{(1)}=e_{2}-e_{0}+\ell D 0, \quad-\frac{\pi}{3} \leq \arg (w) \leq 0 \\
& h^{(2)}=e_{0}-e_{1}+\ell^{\prime} D 0, \quad 0 \leq \arg (w) \leq \frac{\pi}{3}
\end{aligned}
$$

where $\ell$ and $\ell^{\prime}$ are nonnegative integers. It is natural to assume that $\ell^{\prime}=\ell$. The two branes in (5.17) then differ by a permutation. Note that both higgs branch branes in (5.17) have the same asymptotic behavior for the generalized central charge as a function of $w$ :

$$
\left\langle h^{(1,2)} \mid 1\right\rangle \sim 2 \pi i \log w
$$

This is reminiscent of the mirror map for the spacetime non-supersymmetric case. When $\ell=0$ or $\ell^{\prime}=0$, the branes in (5.17) become massless at the orbifold point, which generally signals the breakdown of the string perturbation theory. It is possible however, that the states which appear in the Hilbert space of the orbifold theory have positive $\ell, \ell^{\prime}$.

8 We can solve the soliton equations with the flat metric similarly to what is described in appendix B. The solutions emanating from the $\left(x_{1}, x_{3}\right)=(0,0)$ critical point suggest that the corresponding A-brane surface indeed asymptotes to the quarter planes defining $3\left(\hat{I}_{0}-\hat{I}_{-1}\right)$ and $3\left(\hat{I}_{1}-\hat{I}_{0}\right)$ and the transition between the two happens at $\arg (w)=0$. This is another strong piece of evidence in favor of the picture proposed. 


\section{2. $n=4$}

Our next example is $n=4, p=1$. In this case the orbifold group is $Z_{N=n}=Z_{4}$ and both type 0 and type II theory can be defined. There are $n=4$ choices for the reduced intersection matrix $\tilde{\mathcal{I}}$.

(1) The intersection form corresponds to the continued fraction determined by $n /(n-$ $p) \rightarrow[2,2,2]$.

$$
\tilde{\mathcal{I}}=\left(\begin{array}{ccc}
-2 & 1 & 0 \\
1 & -2 & 1 \\
0 & 1 & -2
\end{array}\right)
$$

This describes spacetime-supersymmetric ALE space. In all other examples the intersection form is determined by the continued fraction determined by $n / p \rightarrow[4]$.

$$
\tilde{\mathcal{I}}=\left(\begin{array}{ccc}
1 & -2 & -1 \\
0 & 1 & -2 \\
1 & 0 & 1
\end{array}\right)
$$

The higgs branch brane is given by

$$
h^{(1)}=e_{s}+2 e_{s+1}+3 e_{s+2}
$$

(3)

$$
\tilde{\mathcal{I}}=\left(\begin{array}{ccc}
0 & 1 & -2 \\
1 & 0 & 1 \\
-2 & 1 & 0
\end{array}\right)
$$

and therefore

$$
h^{(2)}=e_{s}+2 e_{s+1}-e_{s+2}
$$

In fact, $\tilde{\mathcal{I}}$ in $(5.22)$ is the intersection matrix for the type II string, and (5.23) is the unique higgs branch brane in this case.

(4)

$$
\tilde{\mathcal{I}}=\left(\begin{array}{ccc}
1 & 0 & 1 \\
-2 & 1 & 0 \\
1 & -2 & 1
\end{array}\right)
$$

This is the transpose of (5.20). The higgs branch brane is

$$
h^{(3)}=e_{s}-2 e_{s+1}-e_{s+2}
$$

We also claim that in $(2)-(4)$

$$
c_{1}=e_{s+1}, c_{2}=e_{s+2}, \quad \text { or } \quad c_{1}=e_{s+2}, c_{2}=e_{s+1}
$$


The reasoning goes as follows. Below we will see that the generalized central charge for the coulomb branch branes is given by (5.2). This formula satisfies (in a certain angular sector)

$$
\left\langle c_{k} \mid 1\right\rangle \sim w \exp \left(\frac{2 \pi i(k-2)}{n}\right)+\mathcal{O}\left(w^{2}\right), \quad k=1,2
$$

The intersection form implies that $c_{k}$ and $e_{k}$ are possibly related as

$$
\left(\begin{array}{c}
e_{s} \\
e_{s+1}
\end{array}\right)=\left(\begin{array}{cc}
a & b \\
c & d
\end{array}\right)\left(\begin{array}{l}
c_{1} \\
c_{2}
\end{array}\right), \quad a d-b c=1
$$

The consistency with (5.5) implies that in this angular sector $e_{0}=c_{2}, e_{1}=c_{1}$ and, more generally, (5.26). (The alternative solution is $e_{1}=c_{1}, e_{2}=-c_{2}$ implies "negative mass" for the $c_{1}$ brane and therefore should be discarded. It is also not consistent with the formula for the higgs branch brane.)

What happens as the phase of $w$ is varied? The relevant angular sectors are depicted in Fig. 4. There are two critical points (modulo LG symmetry)

$$
\nu=0: \quad\left(x_{1}, x_{3}\right)=\left(\frac{\sqrt{-w}}{2}, \frac{\sqrt{-w}}{2}\right) ; \quad \nu=1: \quad\left(x_{1}, x_{3}\right)=\left(\frac{\sqrt{-w} e^{\frac{\pi i}{4}}}{2}, \frac{\sqrt{-w} e^{\frac{3 \pi i}{4}}}{2}\right)
$$

The choice of sign in the square root does not matter, the difference amounts to the LG symmetry. We choose a fundamental domain to be $0 \leq \arg (-w)<2 \pi$ which maps into $0 \leq \arg (\sqrt{-w})<\pi$. For a given value of $w, R e W_{*}$ has different signs for the two critical points. One critical point contributes a growing exponential, while the other a decaying one. The $w$ plane is divided into four big sectors, which can labeled by $\nu=0,1$ and $s=0,1,2,3$, depending on which critical point contributes a growing exponential to which $\hat{I}_{s}$.

$$
\begin{aligned}
-\frac{\pi}{4}<\arg (w)<\frac{\pi}{4}: & \nu=0, s=2 \\
\frac{\pi}{4}<\arg (w)<\frac{3 \pi}{4}: & \nu=1, s=3 \\
\frac{3 \pi}{4}<\arg (w)<\frac{5 \pi}{4}: & \nu=0, s=0 \\
\frac{5 \pi}{4}<\arg (w)<\frac{7 \pi}{4}: & \nu=1, s=1
\end{aligned}
$$

These big sectors differ from each other by a permutation of fractional branes, in exact analogy to the $n=3$ case. The exponentially decaying solution can be identified unambiguously; the logarithmically growing solution is defined up to an addition of exponentially 


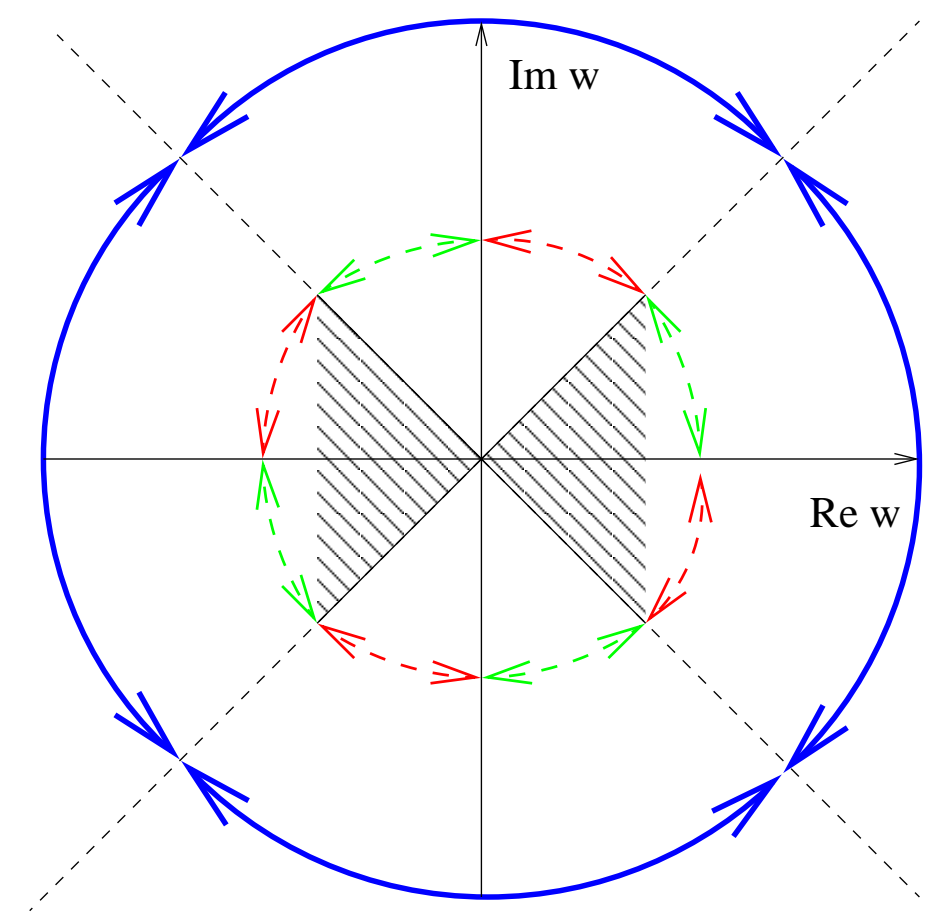

Fig 4. The angular structure in the $w$ plane for $n=4$. When $w$ is in the shaded sectors, the $\nu=0(\nu=1)$ critical point has negative (positive) value of Re W. This is reversed in the unshaded sectors. Big blue arrows mark sectors which differ by permutations of fractional branes. Dashed red and green arrows mark sectors which differ by the type of higgs branch brane

decaying piece:

$$
\begin{array}{rlll}
-\frac{\pi}{4}<\arg (w)<\frac{\pi}{4}: & \hat{I}_{1}-\hat{I}_{3} \sim \frac{\pi i}{2} \log w, & \hat{I}_{1}+\hat{I}_{3}-2 \hat{I}_{0} \sim \exp \left(-W_{1}\right) \\
\frac{\pi}{4}<\arg (w)<\frac{3 \pi}{4}: & \hat{I}_{2}-\hat{I}_{0} \sim \frac{\pi i}{2} \log w, & \hat{I}_{2}+\hat{I}_{0}-2 \hat{I}_{1} \sim \exp \left(-W_{0}\right) \\
\frac{3 \pi}{4}<\arg (w)<\frac{5 \pi}{4}: & \hat{I}_{3}-\hat{I}_{1} \sim \frac{\pi i}{2} \log w, & \hat{I}_{3}+\hat{I}_{1}-2 \hat{I}_{2} \sim \exp \left(-W_{1}\right) \\
\frac{5 \pi}{4}<\arg (w)<\frac{7 \pi}{4}: & \hat{I}_{0}-\hat{I}_{2} \sim \frac{\pi i}{2} \log w, & \hat{I}_{2}+\hat{I}_{0}-2 \hat{I}_{1} \sim \exp \left(-W_{0}\right)
\end{array}
$$

Let us restrict to the big sector $-\frac{\pi}{4}<\arg (w)<\frac{\pi}{4}$. The exponentially decaying solution is given by the first equation in (5.31). According to (5.3), the respective coulomb branch brane $c_{2}$ must be equal to $e_{0}$. Hence, there are two possibilities, consistent with (5.26). Either $c_{1}=e_{1}, c_{2}=e_{0}$ and $s=3$ in (5.26) (remember that $s$ is defined $\bmod n$.) Then, 
$\left\langle c_{2} \mid 1\right\rangle \sim \hat{I}_{1}+\hat{I}_{3}-2 \hat{I}_{0},\left\langle c_{1} \mid 1\right\rangle \sim \hat{I}_{2}+\hat{I}_{0}-2 \hat{I}_{1}$. In this case

$$
\begin{aligned}
& \left\langle h^{(1)} \mid 1\right\rangle \sim 4\left(\hat{I}_{2}-\hat{I}_{1}\right) \sim \exp \left(-W_{0}\right) \\
& \left\langle h^{(2)} \mid 1\right\rangle \sim 4\left(\hat{I}_{1}-\hat{I}_{0}\right) \sim 2 \pi i \log (w) \\
& \left\langle h^{(3)} \mid 1\right\rangle \sim 4\left(\hat{I}_{0}-\hat{I}_{3}\right) \sim 2 \pi i \log (w)
\end{aligned}
$$

The generalized central charge for $h^{(1)}$ has a growing exponential behavior instead of the expected logarithmic behavior. Alternatively, it might happen that $c_{1}=e_{3}, c_{2}=e_{0}, s=2$ in (5.26), and hence $\left\langle c_{1} \mid 1\right\rangle \sim \hat{I}_{0}+\hat{I}_{2}-2 \hat{I}_{3},\left\langle c_{2} \mid 1\right\rangle \sim \hat{I}_{1}+\hat{I}_{3}-2 \hat{I}_{0}$ and

$$
\begin{aligned}
& \left\langle h^{(1)} \mid 1\right\rangle \sim 4\left(\hat{I}_{1}-\hat{I}_{0}\right) \sim 2 \pi i \log (w) \\
& \left\langle h^{(2)} \mid 1\right\rangle \sim 4\left(\hat{I}_{0}-\hat{I}_{3}\right) \sim 2 \pi i \log (w) \\
& \left\langle h^{(3)} \mid 1\right\rangle \sim 4\left(\hat{I}_{3}-\hat{I}_{2}\right) \sim \exp \left(-W_{0}\right)
\end{aligned}
$$

Now it is $h^{(3)}$ whose behavior is not consistent with the expectations.

In the Appendix B we analyze the behavior of the A-brane surfaces. The results suggest that the sector $-\pi / 4 \leq \arg (w) \leq \pi / 4$ is subdivided into the two subsectors, similar to the $n=3$ case. Whenever $0 \leq \arg (w) \leq \pi / 4$, we have $c_{2}=e_{0}, c_{1}=e_{1}$, and $\langle h \mid 1\rangle \sim 4\left(\hat{I}_{1}-\hat{I}_{0}\right)$. That is, in this subsector

$$
h=h^{(2)}=e_{3}+2 e_{0}-e_{1}, \quad 0 \leq \arg (w) \leq \frac{\pi}{4}
$$

Whenever $-\pi / 4 \leq \arg (w) \leq 0$, we have $c_{1}=e_{3}, c_{2}=e_{0}$, and $\langle h \mid 1\rangle \sim 4\left(\hat{I}_{0}-\hat{I}_{3}\right)$. In this subsector we again have

$$
h=h^{(2)}=e_{2}+2 e_{3}-e_{0}, \quad-\frac{\pi}{4} \leq \arg (w) \leq 0
$$

Hence, among the three possible expressions for the higgs branch brane consistent with the intersection form, only one is realized. It is the one which appears in the type II case. [As before, there is a freedom to add D0 branes to (5.34), (5.35)]

In Appendix B we also analyze the shape of the brane emanating from the $\nu=1$ critical point. In the analysis above it has been assumed that in the angular sector $-\pi / 4 \leq$ $\arg (w) \leq \pi / 4$, the corresponding central charge is given by $\langle c \mid 1\rangle \sim \hat{I}_{1}+\hat{I}_{3}-2 \hat{I}_{0}$, since the value of $W$ at the critical point is positive and we expect the generalized central charge to decay exponentially. This is confirmed by solving the soliton equations in Appendix B. 


\section{Discussion}

In this paper we studied A-branes in the LG model which describes the resolution of the spacetime non-supersymmetric $\mathbb{C}^{2} / \mathbb{Z}_{n(p=1)}$ orbifold. The model has coulomb branch branes, supported at the minima of the superpotential $W$ away from the origin, and higgs branch branes, associated with the critical point at the origin. The generalized central charge for these branes, defined in Section 3, satisfies the GKZ equation. This fact, together with a knowledge of the critical points of $W$, and the numerical analysis of the A-brane shape, allows us to compute the central charges for both coulomb and higgs branch branes. The results have the expected asymptotic behavior (determined by the value of $W$ at the corresponding critical point). They are also consistent with the open string Witten index. That is, in the examples that we have studied, we can identify coulomb branch branes and fractional branes using the intersection matrix and first order conformal perturbation theory at the orbifold point. The higgs branch brane is given by a linear combination of fractional branes, so its generalized central charge can be computed accordingly. This procedure gives the same result as integrating (3.3) over the higgs branch brane propeller surface 9 . This result has logarithmic asymptotics as $|w| \rightarrow \infty$, as expected from the form of the integrand at the origin. Similar logarithmic behavior arises also in the spacetime supersymmetric case.

The complex $w$ plane, is divided into $n$ angular sectors, related by the permutation of the fractional branes. Hence, it is the phase of the tachyon VEV that is responsible for a particular ordering of fractional branes being realized. Moreover, each sector is further divided into subsectors, where different expressions for the higgs branch brane are valid.

In writing formulae for the higgs branch brane one must bear in mind that our techniques are not powerful enough to distinguish between $h$ and $h+\ell D 0$ where $\ell \in \mathbb{Z}$ and $D 0=e_{0}+\cdots+e_{n-1}$. Recall that $D 0$ is in the annihilator of the intersection form. In principle this ambiguity could be fixed by determining the constant $C_{*}$ in $\langle h \mid 1\rangle=C_{*}+\sum c_{s} \hat{I}_{s}$ using the propeller surface to determine (3.3). Unfortunately we have not been able to extract this constant. In the case $n=3$ we found the combination $h=e_{2}-e_{0}$ in (5.17) when $\ell=0$. Taken at face value this would be a massless brane at the orbifold point, signaling a breakdown in string perturbation theory. For this reason we find positive values of $\ell$ in (5.17) more likely. Clearly, further work is needed here.

9 Numerical data suggests that the propeller surface depends only weakly on the LG metric: its asymptotics, which are important for the value of the integral, seem to be metric-independent. 
Among the coulomb branch branes, approximately one half have exponentially growing periods while the other half have exponentially decaying periods. The blockdiagonalization of the intersection matrix implies that the coulomb branch branes are decoupled from their higgs branch counterparts. Surprisingly, the two coulomb branch branes in $\mathbb{C}^{2} / \mathbb{Z}_{4(1)}$ are not orthogonal to each other, even though the coulomb vacua are far separated in the IR! It would be interesting to analyze in detail the patterns at higher values of $n$ where many coulomb branch branes associated with critical points with both positive and negative values of $R e W$ are present. This might be useful for the physical interpretation of $(3.3)$ in the non-conformal case.

This work raises a number of technical issues which must be solved in order to make further progress. We used numerical analysis to determine the shape of the propeller branes. The wings depend on the angular sectors in the $w$ plane; the asymptotics change discontinuously as $w$ crosses the borders of these sectors. It would be nice to have some analytic technology to understand these phenomena better. In the $n=4$ case, some wings developed which did not seem to contribute to (3.3). This will probably be a persistent issue for higher $n$, and understanding better the shape of the propeller brane and (3.3) is important for making progress. Similarly, we have made an important assumption that the asymptotics of the propeller branes is independent of the choice of metric $g_{i \bar{j}}$ used in (3.1). We have checked this numerically for the metrics of interest here (see appendix B) but some rigorous results concerning this would be most welcome. Finally, in constructing a map between the fractional branes and the coulomb branch branes, it is would be very useful to know the intersection form for the latter.

Understanding the structure of branes in more general spacetime non-supersymmetric orbifolds is another interesting direction. At present, the higgs branch brane can be expressed in terms of fractional branes for the $\mathbb{C}^{2} / \mathbb{Z}_{n(p)}$ orbifolds which have a resolution in type II theory [7]. Generalizing this result to type 0 theory for arbitrary $n$ and $p$ will probably involve understanding phase diagrams of multiple tachyons, and the space of possible higgs branch branes. It would also be worthwhile understanding spacetime nonsupersymmetric $\mathbb{C}^{3} / \mathbb{Z}_{n}$ orbifolds, where some new features appear already in the closed string sector [21-23].

The Stokes' phenomenon observed in this paper is very likely related to that associated to general semisimple Frobenius manifolds in [24] 225]. It is possible that some of the techniques used in [26] [27] can be applied to elucidate the behavior of generalized periods and their Stokes matrices for general $\mathbb{C}^{2} / \mathbb{Z}_{n(1)}$ orbifolds, or even $\mathbb{C}^{2} / \mathbb{Z}_{n(p)}$ orbifolds. We 
hope our considerations will be useful in understanding homological mirror symmetry for non-Fano manifolds. In the Fano case Stokes' matrices are related to the dimensions of Ext groups of exceptional collections in the derived category of the mirror [26] 227]. (For recent progress in homological mirror symmetry in the Fano case see [28] [29].) In the examples of the $\mathbb{C}^{2} / \mathbb{Z}_{n(p)}$ orbifolds we are instead trying to formulate a quantum version of the McKay correspondence, as explained in [7] 8]. One point which is currently missing is an analogous interpretation of the Stokes' matrices in the non-Fano case.

Stokes' phenomenon has recently played an important role in brane physics in the context of minimal string theory [30]. It is interesting to contrast that application with the present one. In both cases a "brane partition function" satisfies a differential equation with an irregular singular point, and the angular-sector-dependence of exponential growth and decay has important physical consequences. In both cases one can use branes to probe the nature of spacetime, and Stokes' phenomenon has important implications for the resulting spacetime picture. On the other hand, in [30] one works with the non-perturbative brane amplitude (the Baker-Akhiezer function of the matrix model, now also known as the "FZZT partition function") but the present paper only makes use of the perturbative disk one-point function. Nevertheless, there is a common mathematical thread in both examples, since both examples are governed by a family of Landau-Ginzburg theories, and hence by a similar underlying structure of a Frobenius manifold.

In conclusion there is an amazingly rich structure in the D-branes of the $\mathbb{C}^{2} / \mathbb{Z}_{n(1)}$

orbifolds. Surely it is even more intricate in the $\mathbb{C}^{2} / \mathbb{Z}_{n(p)}$ and $\mathbb{C}^{n} / \Gamma$ orbifolds. Elucidating this structure appears to be a challenging project.

Acknowledgements: We would like to thank E. Diaconescu, R. Karp and R. Plesser for discussions. We also thank B. Florea for initial participation in the project and for many useful discussions. This work was supported in part by DOE grant DE-FG02-96ER40949.

\section{Appendix A. Boundary states for fractional branes}

In this appendix we describe in more detail the construction of the boundary states which correspond to fractional branes at the $\mathbb{C}^{2} / \mathbb{Z}_{N}$ orbifold. There are two cases: (1) $p$ 
odd, $N=n$; (2) $p$ even, $N=2 n$. A useful reference is [31. The first step is constructing B-type Ishibashi boundary states

$$
\begin{array}{ll}
\left.\left(\alpha_{m-\nu}-\tilde{\alpha}_{-m+\nu}\right)|s ; \eta\rangle\right\rangle_{N S N S, R R}=0, & m=1,2, \ldots, \quad \nu=\frac{s}{n} \\
\left.\left(\bar{\alpha}_{m+\nu}-\tilde{\bar{\alpha}}_{-m-\nu}\right)|s ; \eta\rangle\right\rangle_{N S N S, R R}=0, & m=0,1, \ldots,
\end{array}
$$

and

$$
\begin{array}{ll}
\left.\left(\psi_{r-\nu}+i \eta \tilde{\psi}_{-r+\nu}\right)|s ; \eta\rangle\right\rangle_{N S N S, R R}=0, & r=\frac{1}{2}+\mathbb{Z}(\mathbb{Z}) \quad \text { for NS(R), } r-\nu \geq 0 \\
\left.\left(\bar{\psi}_{r+\nu}+i \eta \tilde{\bar{\psi}}_{-r-\nu}\right)|s ; \eta\rangle\right\rangle_{N S N S, R R}=0, & r+\nu \geq 0, \quad \eta= \pm 1
\end{array}
$$

One can verify that conditions (A.1) and (A.2) give rise to the B-boundary states of the $\mathcal{N}=(2,2)$ superconformal theory:

$$
\begin{aligned}
\left.\left(G_{r}^{+}+i \eta \tilde{G}_{-r}^{+}\right)|s ; \eta\rangle\right\rangle_{N S N S, R R} & \left.=0, \quad\left(G_{r}^{-}+i \eta \tilde{G}_{-r}^{-}\right)|s ; \eta\rangle\right\rangle_{N S N S, R R}=0 \\
\left.\left(J_{n}+\tilde{J}_{-n}\right)|s ; \eta\rangle\right\rangle_{N S N S, R R} & =0
\end{aligned}
$$

In the open string sector the basic ingredients are the characters with the insertion of the group element $g^{s}$ where $g$ is the generator of $\mathbb{Z}_{n}$

$$
\chi_{N S, R}^{s}\left(q_{o}\right)=\operatorname{tr}_{N S, R} g^{s} q_{o}^{L_{0}-\frac{c}{12}}, \quad \chi_{N S, R}^{(-)^{F} ; s}\left(q_{o}\right)=\operatorname{tr}_{N S, R}(-)^{F} g^{s} q_{o}^{L_{0}-\frac{c}{12}}
$$

Here $s$ runs from 1 to $N$ and $q_{o}=\exp \left(2 \pi i \tau_{o}\right)$. When $p$ is even and $N=2 n$, the number of independent characters can be reduced to $n$ by the following identity

$$
\chi_{N S}^{s+n}\left(q_{o}\right)=\chi_{N S}^{s}\left(q_{o}\right), \quad \chi_{N S}^{(-)^{F} ; s+n}\left(q_{o}\right)=\chi_{N S}^{(-)^{F} ; s}\left(q_{o}\right)
$$

and

$$
\chi_{R}^{s+n}\left(q_{o}\right)=-\chi_{R}^{s}\left(q_{o}\right), \quad \chi_{R}^{(-)^{F} ; s+n}\left(q_{o}\right)=-\chi_{R}^{(-)^{F} ; s}\left(q_{o}\right)
$$

These identities follow from (2.1). In the $N S$ sector $g^{n}=1$, while in the $R$ sector $g^{n}=$ $(-)^{p+1}=-1$. The characters (A.4) have the following modular transformation properties under $\tau_{o} \rightarrow \tau_{c}=-1 / \tau_{o}$ :

$$
\begin{aligned}
\chi_{N S}^{s}\left(q_{o}\right) & =\sigma(s){ }_{N S N S}\left\langle\left\langle s ; \pm\left|q_{c}^{\frac{1}{2}\left(L_{0}+\tilde{L}_{0}-\frac{c}{12}\right)}\right| s \pm\right\rangle\right\rangle_{N S N S} \\
\chi_{N S}^{(-)^{F} ; s}\left(q_{o}\right) & =-\sigma(s)_{R R}\left\langle\left\langle s ; \pm\left|q_{c}^{\frac{1}{2}\left(L_{0}+\tilde{L}_{0}-\frac{c}{12}\right)}\right| s \pm\right\rangle\right\rangle_{R R} \\
\chi_{R}^{s}\left(q_{o}\right) & =\sigma(s){ }_{N S N S}\left\langle\left\langle s ; \pm\left|q_{c}^{\frac{1}{2}\left(L_{0}+\tilde{L}_{0}-\frac{c}{12}\right)}\right| s \mp\right\rangle\right\rangle_{N S N S} \\
\chi_{R}^{(-)^{F} ; s}\left(q_{o}\right) & =-\sigma(s)_{R R}\left\langle\left\langle s ; \pm\left|q_{c}^{\frac{1}{2}\left(L_{0}+\tilde{L}_{0}-\frac{c}{12}\right)}\right| s \mp\right\rangle\right\rangle_{R R}
\end{aligned}
$$


where

$$
\sigma(s)=4 \sin \left(\frac{\pi s}{n}\right) \sin \left(\frac{\pi s p}{n}\right)
$$

With the exception of the factor $\sigma(s)$, the modular transformation properties (A.7) are the same as those of the untwisted characters. The boundary states are constructed by requiring open-closed string duality. Consider $N=n$ case first.

$$
\begin{aligned}
\operatorname{tr}_{a b ; N S} q_{o}^{L_{0}-\frac{c}{12}} & =\frac{1}{n} \sum_{s=0}^{n-1} \omega_{n}^{(a-b) s} \sigma(s)_{N S N S}\left\langle\left\langle s ; \pm\left|q_{c}^{\frac{1}{2}\left(L_{0}+\tilde{L}_{0}-\frac{c}{12}\right)}\right| s \pm\right\rangle\right\rangle_{N S N S} \\
& ={ }_{N S N S}\left\langle a ; \pm\left|q_{c}^{\frac{1}{2}\left(L_{0}+\tilde{L}_{0}-\frac{c}{12}\right)}\right| b ; \pm\right\rangle_{N S N S}
\end{aligned}
$$

where we introduced the Cardy state $|a ; \eta\rangle_{N S N S}$ and $\omega_{n}=\exp (2 \pi i / n)$. Eq. (A.9) and its counterpart with $\operatorname{tr}_{a b ; R} q_{o}^{L_{0}-\frac{c}{12}}$ in the RHS implies

$$
\left.|a ; \eta\rangle_{N S N S}=\sum_{s=0}^{n-1} \omega_{n}^{a s} \sqrt{\sigma(s)}|s ; \eta\rangle\right\rangle_{N S N S}
$$

Similarly,

$$
\left.|a ; \eta\rangle_{R R}=\sum_{s=0}^{n-1} \omega_{n}^{a s} \sqrt{\sigma(s)}|s ; \eta\rangle\right\rangle_{R R}
$$

Type 0 theory admits two types of branes distinguished by the value of $\eta$ :

$$
|a ;+\rangle=\frac{1}{\sqrt{2}}\left(|a ;+\rangle_{N S N S}+|a ;+\rangle_{R R}\right), \quad|a ;-\rangle=\frac{1}{\sqrt{2}}\left(-|a ;-\rangle_{N S N S}+|a ;-\rangle_{R R}\right)
$$

In type II theory, only one combination is invariant under the GSO projection

$$
|a ; I I\rangle=\frac{1}{2}\left(|a ;+\rangle_{N S N S}-|a ;-\rangle_{N S N S}+|a ;+\rangle_{R R}+|a ;-\rangle_{R R}\right)
$$

The situation with $p$ even $n$ odd, where type II can not be defined and the orbifold group is $\mathbb{Z}_{N}=\mathbb{Z}_{2 n}$ is a little bit more tricky. Now $a$ in the open string sector is forced to run from 0 to $2 n-1$ and the analog of (A.9) is

$$
\operatorname{tr}_{a b ; N S} q_{o}^{L_{0}-\frac{c}{12}}=\frac{1}{2 n} \sum_{s=0}^{n-1}\left(1+(-)^{a-b}\right) \omega_{2 n}^{(a-b) s} \sigma(s)_{N S N S}\left\langle\left\langle s ; \pm\left|q_{c}^{\frac{1}{2}\left(L_{0}+\tilde{L}_{0}-\frac{c}{12}\right)}\right| s \pm\right\rangle\right\rangle_{N S N S}
$$

where we converted the sum over $s=0, \ldots, 2 n-1$ to the sum over $s=0, \ldots, n-1$ using (A.5) and $\omega_{2 n}=\exp (2 \pi i / 2 n)$. The closed string sector in type 0 is invariant under $s \rightarrow s+n$, that is why there are $n$ Ishibashi states in (A.14). The sum in (A.14) is zero unless $a-b$ 
is even. This prompts us to introduce two types of branes: the ones with $a=2 a^{\prime} ; \eta=+1$ and the ones with $a=2 a^{\prime}+1 ; \eta=-1$. (Now, in addition to $\mathbb{Z}_{n}$ quantum symmetry which permutes the branes, there is a $\mathbb{Z}_{2}$ symmetry which amounts to multiplying $\eta$ by -1.) The boundary states are

$$
\left.\left|a^{\prime} ;+\right\rangle_{N S N S, R R}=\sum_{s=0}^{n-1} \omega_{n}^{a^{\prime} s} \sqrt{\sigma(s)}|s ;+\rangle\right\rangle_{N S N S, R R}
$$

and

$$
\left.\left|a^{\prime} ;-\right\rangle_{N S N S, R R}=\sum_{s=0}^{n-1} \omega_{n}^{\left(a^{\prime}+\frac{1}{2}\right) s} \sqrt{\sigma(s)}|s ;-\rangle\right\rangle_{N S N S, R R}
$$

These boundary states are consistent with (A.14) and its counterpart with $(-)^{F}$ inserted in the LHS. They are also consistent with the corresponding open string expressions in the $R$ sector:

$$
\operatorname{tr}_{a b ; R} q_{o}^{L_{0}-\frac{c}{12}}=\frac{1}{2 n} \sum_{s=0}^{n-1}\left(1+(-)^{a-b-1}\right) \omega_{2 n}^{(a-b) s} \sigma(s)_{N S N S}\left\langle\left\langle s ;+\left|q_{c}^{\frac{1}{2}\left(L_{0}+\tilde{L}_{0}-\frac{c}{12}\right)}\right| s-\right\rangle\right\rangle_{N S N S}
$$

and

$$
\operatorname{tr}_{a b ; R}(-)^{F} q_{o}^{L_{0}-\frac{c}{12}}=\frac{1}{2 n} \sum_{s=0}^{n-1}\left(1+(-)^{a-b-1}\right) \omega_{2 n}^{(a-b) s} \sigma(s)_{R R}\left\langle\left\langle s ;+\left|q_{c}^{\frac{1}{2}\left(L_{0}+\tilde{L}_{0}-\frac{c}{12}\right)}\right| s-\right\rangle\right\rangle_{R R}
$$

\section{Appendix B. Shape of A-branes}

In this appendix we analyze the shape of A-branes in the LG theory. As explained in 12 these branes are Lagrangian surfaces whose image in the $W$ plane is a semi-infinite real line emanating from the critical point $\phi^{i}=\phi_{*}^{i}$ and going in the positive real direction. More practically, one is instructed to solve the soliton equation (3.1). The shape of the A-brane associated with a given critical point is the set of all trajectories satisfying (3.1). Near the critical point, the set of solutions can be parametrized by a small sphere, as in (3.2).

Let us specialize to our LG model. The superpotential is

$$
W=x_{1}^{n}+w x_{1} x_{3}+x_{3}^{n}
$$


To write the soliton equations we must make a choice of metric. The measure factor in (3.3) (which originates from a path integral) suggests that one should use the metric $d s^{2}=\left|\frac{d x_{1}}{x_{1}}\right|^{2}+\left|\frac{d x_{3}}{x_{3}}\right|^{2}$ for large $x_{i}$. This metric is inconvenient for displaying the results of the numerical analysis because the soliton equations develop singularities at finite values of $\sigma$. We have instead used the metric $d s^{2}=\left|x_{1} d x_{1}\right|^{2}+\left|x_{3} d x_{3}\right|^{2}$ because this is the simplest metric for which there is no singularity at finite $\sigma$. A tedious numerical check shows that the asymptotics of the propeller branes described below is in fact independent of the choice of the metric. It would be very useful to establish this rigorously. Some further comments on metric dependence can be found below.

The soliton equations are

$$
\frac{d x_{1}}{d \sigma}=\frac{1}{\left|x_{1}\right|^{2}}\left(n \bar{x}_{1}^{n-1}+\bar{w} \bar{x}_{3}\right), \quad \frac{d x_{3}}{d \sigma}=\frac{1}{\left|x_{3}\right|^{2}}\left(n \bar{x}_{3}^{n-1}+\bar{w} \bar{x}_{1}\right)
$$

The non-zero critical points are given by (4.25) and the value of $W_{*}$ by (4.26). Substituting $x_{1}=x_{1}^{(\nu)}+\delta x_{1}, x_{3}=x_{3}^{(\nu)}+\delta x_{3}$ we have

$$
W=W_{\nu}+\frac{w}{2}\left(e^{-\frac{2 \pi i \nu}{n}}(1-n) \delta x_{1}^{2}+2 \delta x_{1} \delta x_{3}+e^{\frac{2 \pi i \nu}{n}}(1-n) \delta x_{3}^{2}\right)
$$

B.1. $n=3$

For $n=3$ there is a single critical point with $\nu=0, x_{1}=x_{3}=-w / 3$ and $W_{0}=w^{3} / 27$. The change of coordinates which diagonalizes $(\mathbb{B . 3})$ is

$$
u=\frac{\delta x_{1}+\delta x_{3}}{\sqrt{2}}, \quad v=\frac{\delta x_{1}-\delta x_{3}}{\sqrt{2}}
$$

which recasts $(\mathrm{B} .3)$ as

$$
W=W_{\nu}+|w| \exp \left(i \varphi_{w}+i \pi\right)\left(\frac{1}{2} u^{2}+\frac{3}{2} v^{2}\right)
$$

where we introduced $\varphi_{w}=\arg (w)$. The wavefront is a circle and can be parameterized by a single angle $\theta$

$$
\begin{aligned}
& x_{1}=-\frac{w}{3}+\frac{\epsilon}{\sqrt{|w|}} e^{-i \frac{\varphi_{w}+\pi}{2}}\left(\cos \theta+\frac{1}{\sqrt{3}} \sin \theta\right) \\
& x_{3}=-\frac{w}{3}+\frac{\epsilon}{\sqrt{|w|}} e^{-i \frac{\varphi_{w}+\pi}{2}}\left(\cos \theta-\frac{1}{\sqrt{3}} \sin \theta\right)
\end{aligned}
$$


When $x_{1}, x_{3}$ are large, the $w x_{1} x_{3}$ term in the superpotential (B.1) is negligible. Therefore in this regime the equations for $x_{1}$ and $x_{3}$ decouple and the A-brane degenerates to the product of lines $x_{1}^{n} \in \mathbb{R}_{+}$and $x_{3}^{n} \in \mathbb{R}_{+}$, which are used to define $\hat{I}$ 's. Each of these surfaces is essentially a quarter plane. There are $n^{2}$ choices of defining lines, but the LG symmetry brings it down to $n$ inequivalent surfaces. Generally, a circle parametrized by $\theta$ splits into several components; at large worldsheet time $\sigma$, each component traces some quarter plane described above. Therefore the shape of the A-brane surfaces resembles that of a propeller, and we call these surfaces "propeller branes". The integral of (3.3) over each wing of the propeller defines a function $\hat{I}_{s}$.

By solving the soliton equations (B.2) with the initial conditions (B.6) we can determine which combination of $\hat{I}$ 's corresponds to a given coulomb branch brane. The relevant differential equation can be solved by Mathematica. In Fig. 5,6 we present a plot of $\arg \left(x_{1}\right), \arg \left(x_{3}\right)$ as functions of $\sigma$ for several values of $\theta$.

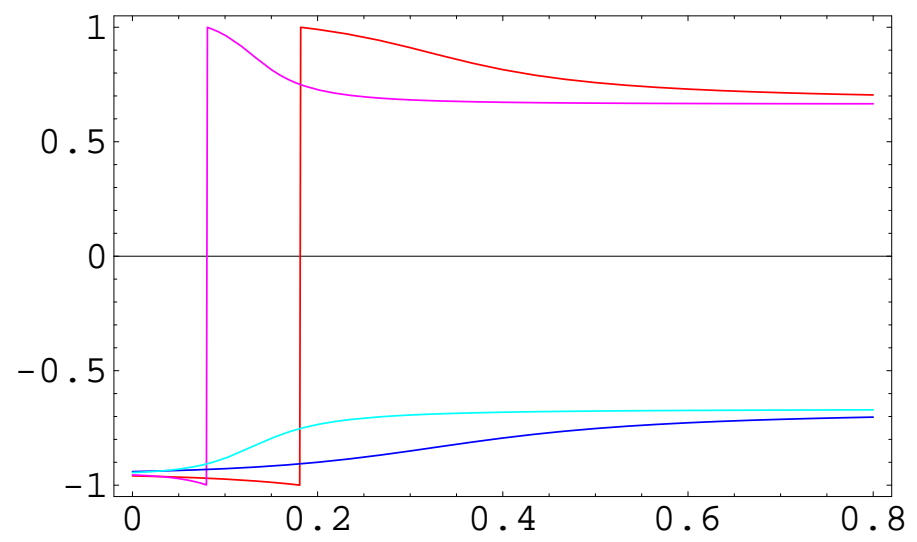

Fig 5. Solution of soliton equation. Horizontal axis corresponds to $\sigma$. Vertical axis is $\arg \left(x_{1}(\sigma)\right) / \pi$. The lines are computed for $w=\exp (0.05 \pi i), \theta=0$ (orange), $\pi / 2$ (pink), $3 \pi / 2$ (turquoise), $\pi$ (blue). They asymptote to $\pm 2 / 3$ as $\sigma \rightarrow \infty$.

From Figs. 5,6 we infer that the pink [turquoise] line which corresponds to $\theta=$ $\pi / 2[\theta=3 \pi / 2]$ asymptotes to $\left(\arg \left(x_{1}\right), \arg \left(x_{3}\right)\right)=(2 \pi / 3,-2 \pi / 3)\left[\left(\arg \left(x_{1}\right), \arg \left(x_{3}\right)\right)=\right.$ $(-2 \pi / 3,2 \pi / 3)$.] For $e^{i \theta}$ in most of the upper (lower) half plane, the asymptotics are similar to those of $\theta=\pi / 2(\theta=3 \pi / 2)$. These two regions define two different representatives of $\hat{I}_{0}$ related by the LG symmetry. For $\theta \approx 0$ the asymptotics corresponds to $\hat{I}_{1}$, while for 


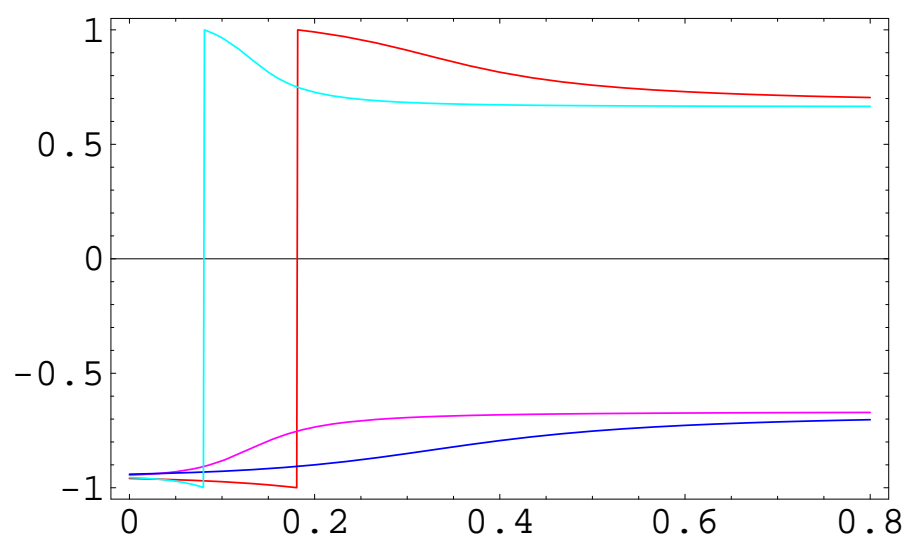

Fig 6. Solution of soliton equation. Horizontal axis corresponds to $\sigma$. Vertical axis $i s \arg \left(x_{3}(\sigma)\right) / \pi$. The lines are computed for $w=\exp (0.05 \pi i), \theta=0$ (orange), $\pi / 2$ (pink), $3 \pi / 2$ (turquoise), $\pi$ (blue). They asymptote to $\pm 2 / 3$ as $\sigma \rightarrow \infty$.

$\theta \approx \pi$ we get $\hat{I}_{-1}$. Hence, we observe that the behavior of solutions is consistent with the identification

$$
\langle c \mid 1\rangle \sim \hat{I}_{1}+\hat{I}_{-1}-2 \hat{I}_{0}, \quad-\pi / 3 \leq \arg (w) \leq \pi / 3
$$

In fact, Figs. 5,6 do not qualitatively change in the whole "big" angular sector $-\pi / 3 \leq$ $\arg (w) \leq \pi / 3$. The qualitative change happens when $w$ crosses the lines $\arg (w)=-\pi / 3$ and $\arg (w)=\pi / 3$. The asymptotics of the coulomb branch brane changes in accord with the rule proposed above: multiplying $w$ by $\exp (2 \pi i / 3)$ (rotating the "big" sector) corresponds to the permutation of the fractional branes.

In the discussion above we determined that the integral (3.3) over the propeller surface originating at the coulomb branch critical point gives rise to (B.7). This is because the propeller surface in question has four wings. Integrating (3.3) over these wings gives rise to $I_{1}, I_{-1}$ and $I_{0}$ (twice). Eq. (B.7) is then consistent with (4.20), with the one-point function for the fractional brane, and with the intersection matrix. Nevertheless it is desirable to have an independent way of determining the orientation of the wings, i.e. the signs in eq. (B.7). Consider a wing defined by $\left(x_{1}, x_{3}\right)=\left(e^{\frac{2 \pi i s_{1}}{n}} t_{1}, e^{\frac{2 \pi i s_{3}}{n}} t_{3}\right)$ for large positive $t_{1}$ and $t_{3}$. A soliton trajectory originating at a given value of $\theta$ gives rise to a ray in $\left(t_{1}, t_{3}\right)$ plane. In the simplest scenario, the slope of this ray $\gamma=\left|x_{1}\right| /\left|x_{3}\right|$ is a monotonic function of $\theta$. The orientation is then determined by the sign of $d \gamma / d \theta$. In Fig. 7 we plot $\gamma(\theta)$. There are four regions where this function is monotonic; it takes all values between zero and infinity. 


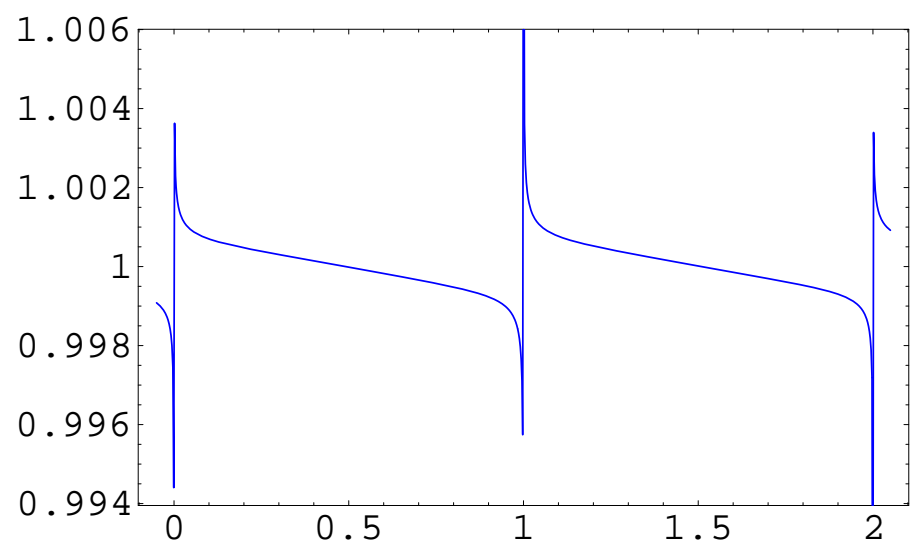

Fig 7. Orientation of the wings $(n=3)$ is determined by $\gamma(\theta)$. Vertical axis is $\gamma \equiv$ $\left|x_{1}\right| /\left|x_{3}\right|$. Horizontal axis is $\theta / \pi$.

These four regions correspond to $I_{1}, I_{-1}$ and $I_{0}$ (twice). The signs of $d \gamma / d \theta$ are consistent with the signs that appear in (B.7).

It is also interesting to investigate the dependence of the solutions of soliton equations on the metric. One natural metric to try is the Euclidean metric, leading to soliton equations of the form

$$
\frac{d x_{1}}{d \sigma}=\left(n \bar{x}_{1}^{n-1}+\bar{w} \bar{x}_{3}\right), \quad \frac{d x_{3}}{d \sigma}=\left(n \bar{x}_{3}^{n-1}+\bar{w} \bar{x}_{1}\right)
$$

We analyzed the solutions of these equations emanating from a nonzero critical point. The technical difference with (B.2) is that now $x_{1}$ or $x_{3}$ run off to infinity at finite values of $\sigma$. Moreover, for generic values of $\theta$ only one of $x_{1}, x_{3}$ runs off to infinity, and only for isolated values of $\theta$ both of them do so. All of this makes eqs. (B.8) more difficult to study numerically then eqs. (B.2). The numerical data suggests that solutions of (B.8) asymptote to the surface that is homologous at the infinity to the one defined by $(\overline{B .2})$. We have also carried out similar checks of metric independence for the metric $d s^{2}=\left|\frac{d x_{1}}{x_{1}}\right|^{2}+\left|\frac{d x_{3}}{x_{3}}\right|^{2}$ (this metric is the natural one to expect from the derivation of the LG theory via $T$-duality). All this suggests that the behavior of the A-brane surface depends only weakly on the metric, and the asymptotics might stay the same for a large class of metric deformations. It is now natural to try to analyze the behavior of the higgs branch brane as well. The metric near the origin is not known, but if the asymptotics do not depend on it, we might as well use eqs. (B.8). The results confirm the picture described in the main text. Restricting to 
the big angular sector $-\pi / 3<\arg (w)<\pi / 3$, the solutions experience an abrupt change as $w$ crosses the line $\arg (w)=0$. Above this line, the solutions asymptote to the surface defining $3\left(\hat{I}_{1}-\hat{I}_{0}\right)$, and below the line to $3\left(\hat{I}_{0}-\hat{I}_{-1}\right)$.

\section{B.2. $n=4$}

Consider now $n=4$. We will restrict our analysis to the angular sector defined by $-\pi / 4<\arg (w)<\pi / 4$ (see Fig. 4). Other big sectors are related to this one by the LG symmetry. We studied the surface corresponding to the higgs branch brane (with the flat metric). The result is

$$
\begin{aligned}
0<\arg (w)<\frac{\pi}{4}: & \langle h \mid 1\rangle \sim 4\left(\hat{I}_{1}-\hat{I}_{0}\right) \\
-\frac{\pi}{4}<\arg (w)<0: & \langle h \mid 1\rangle \sim 4\left(\hat{I}_{3}-\hat{I}_{0}\right)
\end{aligned}
$$

Consider now the contribution of the critical point with $\nu=0, x_{1}=x_{3}=\sqrt{-w} / 2$, $W=-2(w / 2)^{2}$. (We denote the corresponding coulomb branch brane by $c_{1}$ ). As explained before, it contributes a growing exponential, hence it is necessary to solve the soliton equations to determine the asymptotics. Note that in the unshaded sectors in Fig. 4 this critical point contributes a decaying exponential. The integral (3.3) over the coulomb branch branes associated with it, should give rise to the following overlaps:

$$
\begin{aligned}
\frac{\pi}{4}<\arg (w)<\frac{3 \pi}{4}: & & \left\langle c_{1} \mid 1\right\rangle \sim \hat{I}_{2}+\hat{I}_{0}-2 \hat{I}_{1} \\
\frac{5 \pi}{4}<\arg (w)<\frac{7 \pi}{4}: & & \left\langle c_{1} \mid 1\right\rangle \sim \hat{I}_{0}+\hat{I}_{2}-2 \hat{I}_{3}
\end{aligned}
$$

Returning back to the soliton equations, the change of variable which diagonalizes (B.3) is

$$
u=e^{\frac{i \varphi_{w}+i \pi}{2}}\left(\delta x_{1}+\delta x_{3}\right), \quad v=e^{\frac{i \varphi_{w}+i \pi}{2}}\left(\delta x_{1}-\delta x_{3}\right)
$$

which gives rise to

$$
W=W_{0}+\frac{|w|}{2}\left(u^{2}+2 v^{2}\right)
$$

The wavefront near the critical point is

$$
\begin{aligned}
& x_{1}=\frac{\sqrt{-w}}{2}+\frac{\epsilon}{\sqrt{|w|}} e^{-i \frac{\varphi_{w}+\pi}{2}}\left(\cos \theta+\frac{1}{\sqrt{2}} \sin \theta\right) \\
& x_{3}=\frac{\sqrt{-w}}{2}+\frac{\epsilon}{\sqrt{|w|}} e^{-i \frac{\varphi_{w}+\pi}{2}}\left(\cos \theta-\frac{1}{\sqrt{2}} \sin \theta\right)
\end{aligned}
$$


The solutions exhibit more complicated behavior than what we have seen so far. As mentioned above, whenever $w$ is in the unshaded sector, we expect the integral (3.3) over the wings of the propeller surface to give (B.10). This is indeed what the numerical analysis tells us. Suppose $\pi / 4 \leq \arg (w) \leq 3 \pi / 4$. Then integrating (3.3) over the wings gives rise to the first equation of $(\overline{\mathrm{B} .10})$. Crossing the line $\arg (w)=\pi / 4$ into the shaded sector does not change the solution. Figs. 8-10 contain the graphs of $\arg \left(x_{1}\right), \arg \left(x_{3}\right)$ as functions of $\theta$ for large worldsheet time $\sigma$. According to Figs. 8-10, the propeller surface emanating from the $\nu=0$ critical point has four wings. Integrating (3.3) over these wings gives rise to the first equation in $(\mathbb{B . 1 0})$. (This is similar to the $n=3$ case)

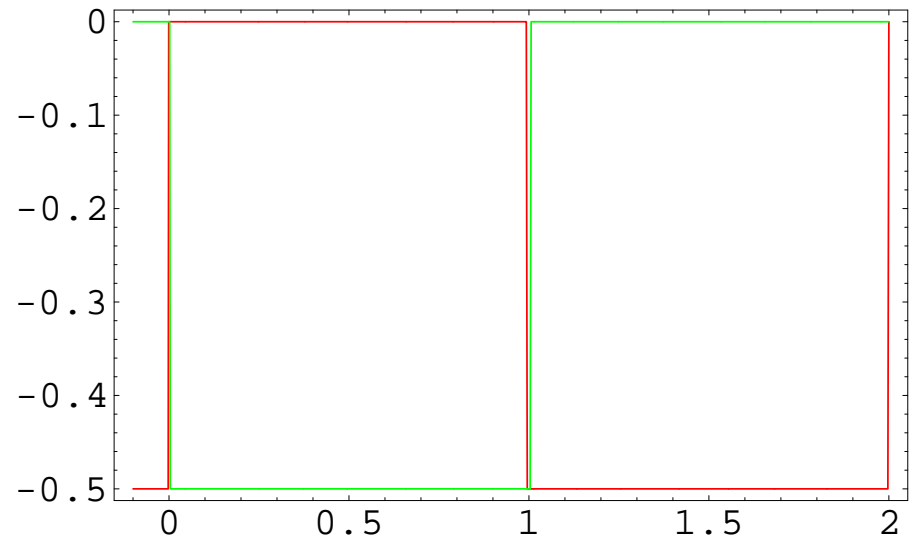

Fig 8. Solution of soliton equation at $\epsilon=0.005, \sigma=8, w=w^{0.9 \pi i / 4}$. Horizontal axis is $\theta / \pi$. Vertical axis is $\arg \left(x_{i}(\sigma)\right) / \pi$. Red line is $i=1$. Green line $i=3$.

To determine the orientation, we compute $\gamma(\theta)$ for large worldsheet time $\sigma$, where $\gamma \equiv\left|x_{1}\right| /\left|x_{3}\right|$, as before. The result is presented in Fig. 11. It is essentially equivalent to the one in Fig. 7, as is consistent with the signs in (B.10).

This is not yet the end of the story, as we will see shortly. As $\arg (w)$ decreases past $\arg (w) \sim 0.6 \pi / 4$, the form of the graphs in Fig. 8 qualitatively changes. Fig. 12 provides an illustration for $\arg (w)=0.45 \pi / 4$, and Figs. 13, 14 give the close-up views.

Figs. 12-14 seem to suggests that the propeller surface develops two extra wings. The integral (3.3) over these wings can cancel, so Figs. 12-14 are still consistent with the expression for the central charge in $(\mathbb{B} .10)$. Yet, they are also consistent with $\langle 1 \mid c\rangle \sim$ 


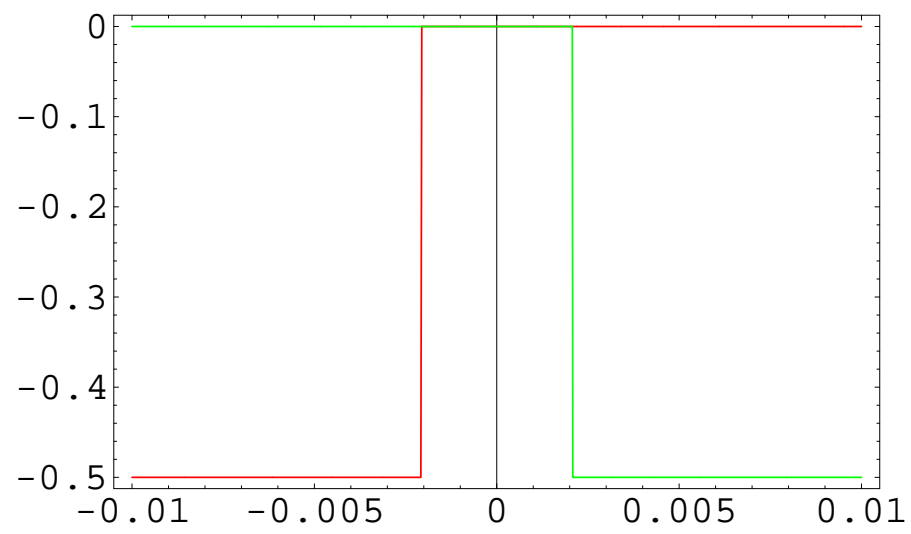

Fig 9. Close-up view of Fig. 8

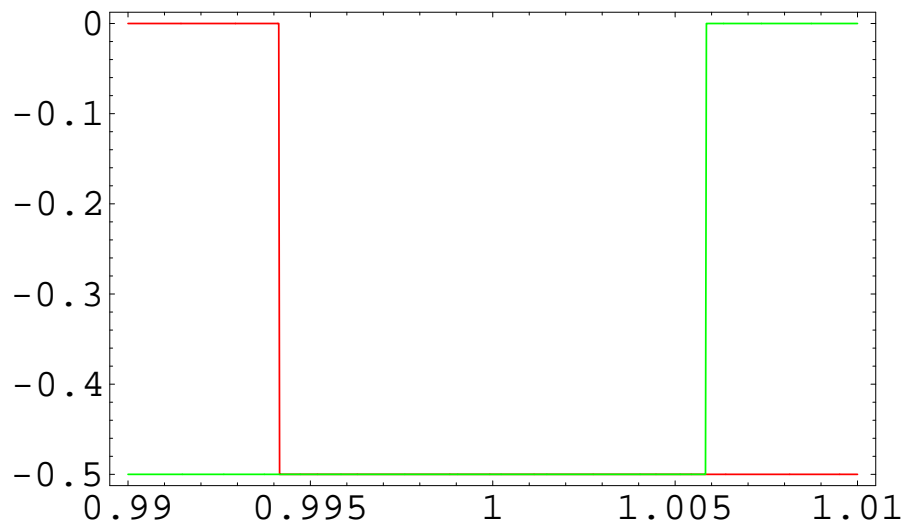

Fig 10. Another close-up view of Fig. 8

$\hat{I}_{2}+2 \hat{I}_{1}-3 \hat{I}_{0}$ [D To resolve this ambiguity, we look again at the $\gamma(\theta)$ graph which determines the orientation. Corresponding graphs are presented in Figs. 15-17.

From Figs. 15-17 we immediately see that the orientation of the four wings which appeared in Fig. 6 does not change as $\arg (w)$ is decreased. Therefore, we must associate $\left\langle c_{1} \mid 1\right\rangle \sim \hat{I}_{2}+\hat{I}_{0}-2 \hat{I}_{1}$, as before. The integrals over the new wings must cancel each other, instead of giving an extra $\hat{I}_{0}$. A similar situation happens for $\arg (w)<0$.

10 Note that as $\arg (w) \rightarrow 0$, the wings which give rise to $\hat{I}_{1}$ shrink but do not disappear until $w$ crosses the $\arg (w)=0$ line. On the contrary, the new wings, which may define extra $2 I_{0}$ disappear $\operatorname{as} \arg (w)$ is increased over $\sim 0.6 \pi / 4$. 


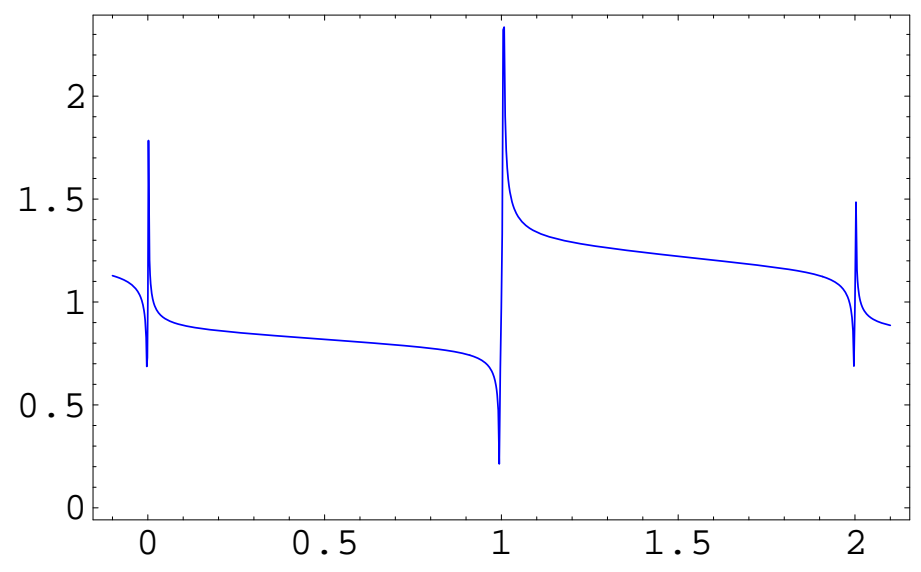

Fig 11. Orientation of the wings $\left(n=4 ; w=e^{0.9 \pi i / 4}\right)$ is determined by $\gamma(\theta)$. Vertical axis is $\gamma \equiv\left|x_{1}\right| /\left|x_{3}\right|$. Horizontal axis is $\theta / \pi$.

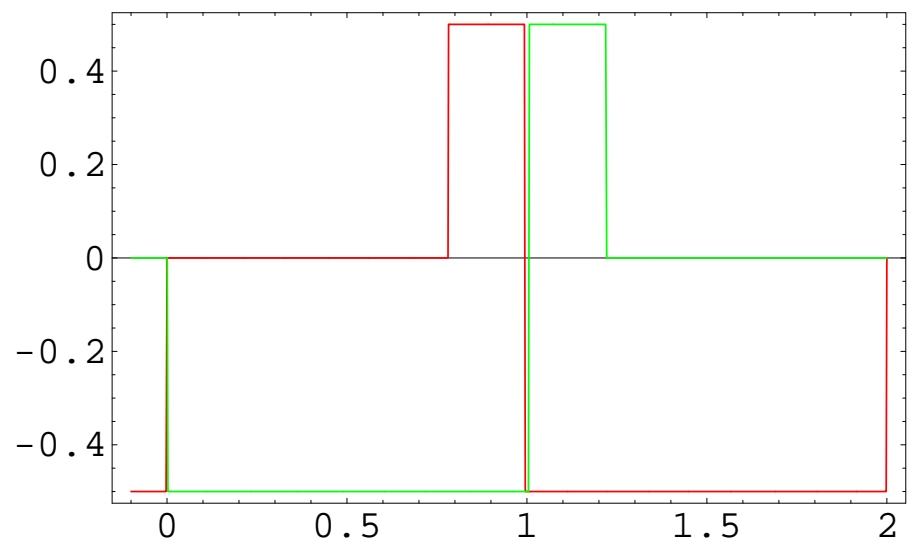

Fig 12. Solution of soliton equation at $\epsilon=0.005, \sigma=8, w=e^{0.45 \pi i / 4}$. Horizontal axis is $\theta / \pi$. Vertical axis is $\arg \left(x_{i}(\sigma)\right) / \pi$. Red line is $i=1$. Green line $i=3$.

To summarize, in the sector $-\pi / 4 \leq \arg (w) \leq \pi / 4$ we have [compare with (B.10)]

$$
\begin{aligned}
0<\arg (w)<\frac{\pi}{4}: & \left\langle c_{1} \mid 1\right\rangle & \sim \hat{I}_{2}+\hat{I}_{0}-2 \hat{I}_{1} \\
-\frac{\pi}{4}<\arg (w)<0: & \left\langle c_{1} \mid 1\right\rangle & \sim \hat{I}_{0}+\hat{I}_{2}-2 \hat{I}_{3}
\end{aligned}
$$

This implies

$$
\begin{aligned}
0<\arg (w)<\frac{\pi}{4}: & c_{2}=e_{0} ; c_{1}=e_{1} \\
-\frac{\pi}{4}<\arg (w)<0: & c_{2}=e_{0} ; c_{1}=e_{3}
\end{aligned}
$$




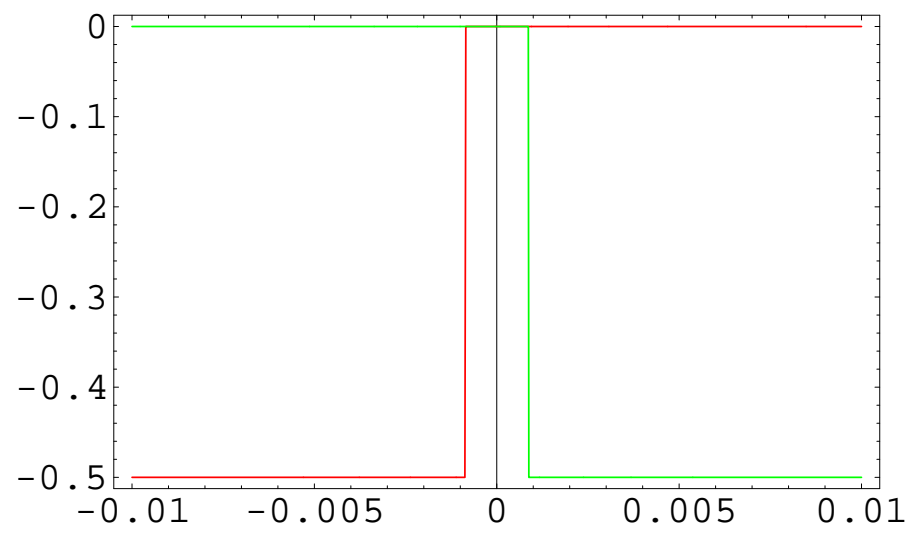

Fig 13. Close-up view of Fig. 12

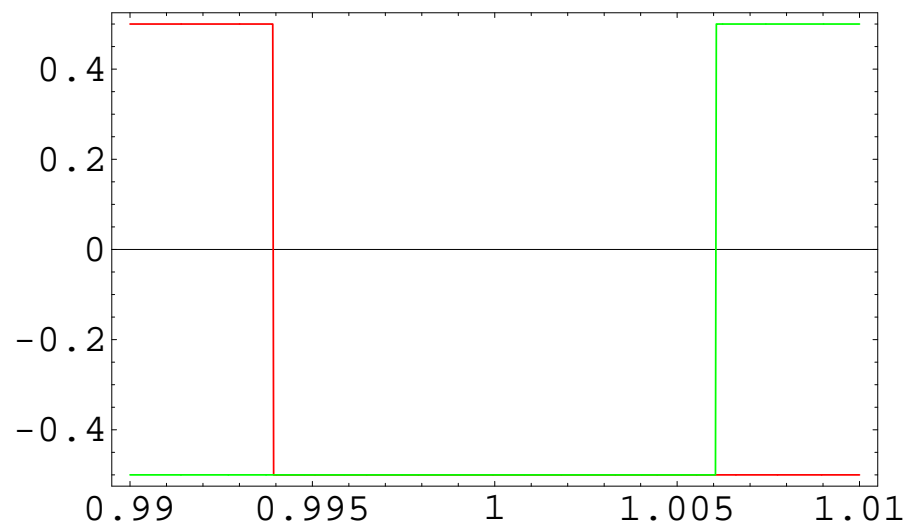

Fig 14. Another close-up view of Fig. 12

Consider now the critical point labeled by $\nu=1$, giving rise to the coulomb branch brane denoted by $c_{2}$. The choice of coordinates which diagonalizes (B.3) is now given by

$$
u=e^{\frac{i \varphi_{w}}{2}+\frac{i \pi}{4}}\left[\frac{1}{2}\left(\frac{3}{\sqrt{2}}-2\right)^{1 / 2} \delta x_{3}-\frac{i}{2}\left(\frac{3}{\sqrt{2}}+2\right)^{1 / 2} \delta x_{1}\right]
$$

and

$$
v=e^{\frac{i \varphi_{w}}{2}+\frac{i \pi}{4}}\left[\frac{1}{2}\left(\frac{3}{\sqrt{2}}+2\right)^{1 / 2} \delta x_{3}-\frac{i}{2}\left(\frac{3}{\sqrt{2}}-2\right)^{1 / 2} \delta x_{1}\right]
$$

which corresponds to

$$
W=W_{1}+\sqrt{2}|w|\left(u^{2}+v^{2}\right)
$$




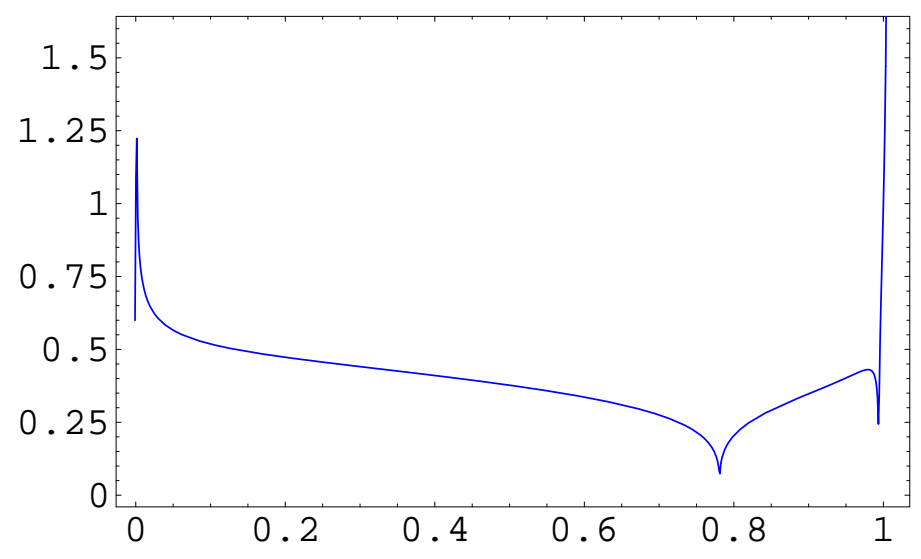

Fig 15. Orientation of the wings ( $\left.n=4 ; w=e^{0.45 \pi i / 4}\right)$ is determined by $\gamma(\theta)$. Vertical axis is $\gamma \equiv\left|x_{1}\right| /\left|x_{3}\right|$. Horizontal axis is $\theta / \pi$.

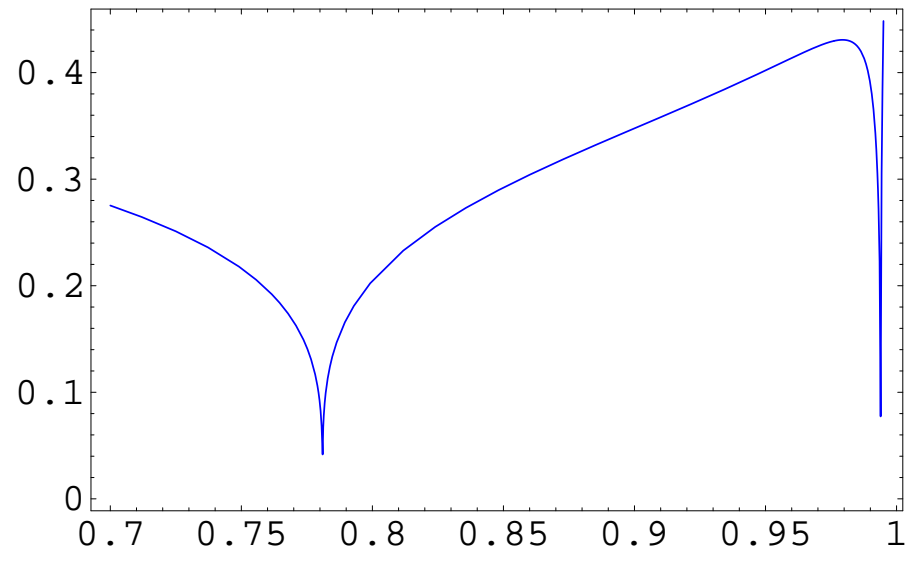

Fig 16. Close-up view of Fig. 15.

The wavefront near the critical point is

$$
\begin{aligned}
& x_{1}=\frac{\sqrt{-w}}{2} e^{\frac{\pi i}{4}}+\frac{\epsilon}{\sqrt{|w|}} e^{-\frac{i \varphi_{w}}{2}-\frac{i \pi}{4}}\left[\frac{i}{2}\left(\frac{3}{\sqrt{2}}+2\right)^{1 / 2} \cos \theta-\frac{i}{2}\left(\frac{3}{\sqrt{2}}-2\right)^{1 / 2} \sin \theta\right] \\
& x_{3}=\frac{\sqrt{-w}}{2} e^{\frac{3 \pi i}{4}}+\frac{\epsilon}{\sqrt{|w|}} e^{-\frac{i \varphi_{w}}{2}-\frac{i \pi}{4}}\left[-\frac{1}{2}\left(\frac{3}{\sqrt{2}}-2\right)^{1 / 2} \cos \theta+\frac{1}{2}\left(\frac{3}{\sqrt{2}}+2\right)^{1 / 2} \sin \theta\right]
\end{aligned}
$$

In the angular sector $-\pi / 4 \leq \arg (w) \leq \pi / 4$, the propeller surface has four wings, and the integral (3.3) reduces to $\left\langle c_{2} \mid 1\right\rangle \sim \hat{I}_{1}+\hat{I}_{3}-2 \hat{I}_{0}$. This is the unique exponentially decaying 


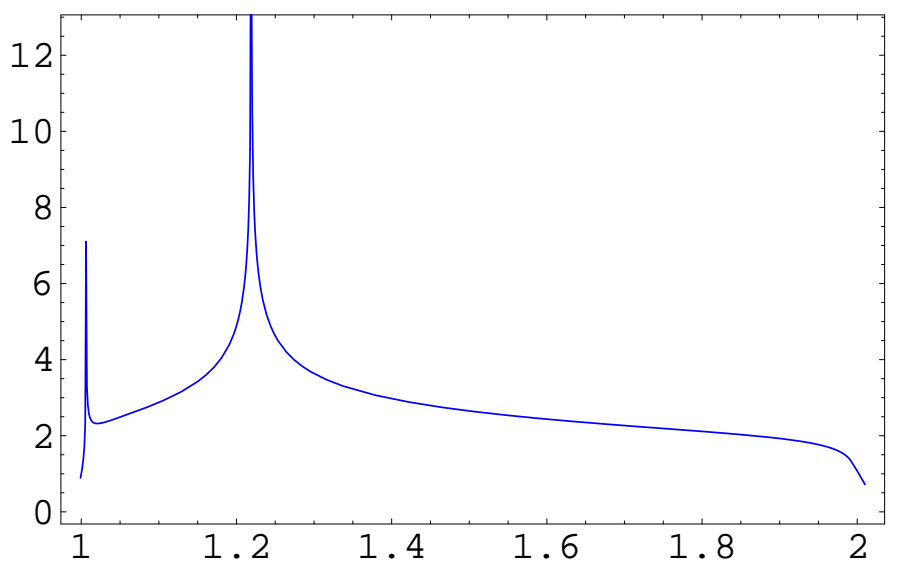

Fig 17. Orientation of the wings $\left(n=4 ; w=e^{0.45 \pi i / 4}\right)$ is determined by $\gamma(\theta)$. Vertical axis is $\gamma \equiv\left|x_{1}\right| /\left|x_{3}\right|$. Horizontal axis is $\theta / \pi$.

solution in this sector. This result is consistent with the fact that the critical value of $W$ at the $\nu=1$ critical point is positive, so we expect the corresponding central charge to decay exponentially. 


\section{References}

[1] A. Adams, J. Polchinski and E. Silverstein, "Don't panic! Closed string tachyons in ALE space-times," JHEP 0110, 029 (2001) arXiv:hep-th/0108075.

[2] E. J. Martinec, "Defects, decay, and dissipated states," arXiv:hep-th/0210231.

[3] M. Headrick, S. Minwalla and T. Takayanagi, "Closed string tachyon condensation: An overview," Class. Quant. Grav. 21, S1539 (2004) arXiv:hep-th/0405064.

[4] J. A. Harvey, D. Kutasov, E. J. Martinec and G. Moore, "Localized tachyons and RG flows," arXiv:hep-th/0111154.

[5] J. A. Harvey, D. Kutasov and E. J. Martinec, "On the relevance of tachyons," arXiv:hep-th/0003101.

[6] C. Vafa, "Mirror symmetry and closed string tachyon condensation," arXiv:hepth/0111051.

[7] E. J. Martinec and G. Moore, "On decay of K-theory," arXiv:hep-th/0212059.

[8] G. W. Moore and A. Parnachev, "Localized tachyons and the quantum McKay correspondence," JHEP 0411, 086 (2004) arXiv:hep-th/0403016.

[9] E. Witten, "Phases of $\mathrm{N}=2$ theories in two dimensions," Nucl. Phys. B 403, 159 (1993) hep-th/9301042.

[10] D. R. Morrison and M. Ronen Plesser, "Summing the instantons: Quantum cohomology and mirror symmetry in toric varieties," Nucl. Phys. B 440, 279 (1995) hepth/9412236.

[11] I. V. Melnikov and M. R. Plesser, "The Coulomb branch in gauged linear sigma models," arXiv:hep-th/0501238; I. V. Melnikov and M. R. Plesser, "A-Model Correlators from the Coulomb Branch," arXiv:hep-th/0507187.

[12] K. Hori and C. Vafa, "Mirror symmetry," arXiv:hep-th/0002222; K. Hori, A. Iqbal and C. Vafa, "D-branes and mirror symmetry," arXiv:hep-th/0005247;

[13] L. J. Dixon, D. Friedan, E. J. Martinec and S. H. Shenker, "The Conformal Field Theory Of Orbifolds," Nucl. Phys. B 282, 13 (1987).

[14] D. R. Morrison and M. Ronen Plesser, "Towards mirror symmetry as duality for two dimensional abelian gauge theories," Nucl. Phys. Proc. Suppl. 46, 177 (1996) arXiv:hep-th/9508107.

[15] M. R. Douglas and B. Fiol, "D-branes and discrete torsion. II," arXiv:hep-th/9903031.

[16] M. Berkooz and M. R. Douglas, "Five-branes in M(atrix) theory," Phys. Lett. B 395, 196 (1997) arXiv:hep-th/9610236.

[17] M. Berkooz, M. R. Douglas and R. G. Leigh, "Branes intersecting at angles," Nucl. Phys. B 480, 265 (1996) [arXiv:hep-th/9606139.

[18] D. Kaminski and R.B. Paris, "Asymptotics of a class of multidimensional Laplace-type integrals. I. Double integrals," Phil. Trans. R. Soc. London. A (1998)356583 
[19] R.B. Dingle, Asymptotic Expansions: their derivation and interpretation, Academic Press, 1973

[20] W. Balser, W.B. Jurkat, D.A. Lutz, Birkhoff Invariants and Stokes' Multipliers for Meromorphic Linear Differential Equations, Journal of Math. Analysis and Applications, 71(1979)48-94

[21] D. R. Morrison, K. Narayan and M. R. Plesser, "Localized tachyons in C(3)/Z(N)," JHEP 0408, 047 (2004) arXiv:hep-th/0406039.

$[22] \quad T$. Sarkar, "On localized tachyon condensation in $\mathrm{C}^{* *} 2 / \mathrm{Z}(\mathrm{n})$ and $\mathrm{C}^{* *} 3 / \mathrm{Z}(\mathrm{n})$," Nucl. Phys. B 700, 490 (2004) [arXiv:hep-th/0407070].

[23] D. R. Morrison and K. Narayan, "On tachyons, gauged linear sigma models, and flip transitions," JHEP 0502, 062 (2005) arXiv:hep-th/0412337.

[24] B. Dubrovin, "Painleve' transcendents and two-dimensional topological field theory," arXiv:math.AG/9803107

[25] B. Dubrovin, "Geometry and analytic theory of Frobenius manifolds," arXiv:math .AG/9807034

[26] D. Guzzetti, "Stokes matrices and monodromy of the quantum cohomology of projective spaces," arXiv:math.AG/9904099

[27] K.Ueda, "Stokes matrices for the quantum cohomologies of Grassmannians," arXiv: math.AG/0503355

[28] D. Auroux, L. Katzarkov, and D. Orlov, "Mirror symmetry for weighted projective planes and their noncommutative deformations," arXiv:math.AG/0404281

[29] D. Auroux, L. Katzarkov, and D. Orlov, "Mirror symmetry for Del Pezzo surfaces: vanishing cycles and coherent sheaves," arXiv:math.AG/0506166

[30] J. Maldacena, G. W. Moore, N. Seiberg and D. Shih, "Exact vs. semiclassical target space of the minimal string," JHEP 0410, 020 (2004) arXiv:hep-th/0408039.

[31] M. Billo, B. Craps and F. Roose, "Orbifold boundary states from Cardy's condition," JHEP 0101, 038 (2001) arXiv:hep-th/0011060. 